\title{
Cosmic evolution of the galaxy's mass and luminosity functions by morphological type from multi-wavelength data in the CDF-South
}

\author{
A. Franceschini ${ }^{1}$, G. Rodighiero ${ }^{1}$, P. Cassata ${ }^{1}$, S. Berta ${ }^{1}$, M. Vaccari ${ }^{1,2}$, M. Nonino ${ }^{3}$, E. Vanzella ${ }^{3}$, E. Hatziminaoglou ${ }^{4}$, \\ J. Antichi ${ }^{1,5}$, and S. Cristiani ${ }^{3}$
}

1 Dipartimento di Astronomia, Università di Padova, Vicolo Osservatorio 2, 35122 Padova, Italy e-mail: franceschini@pd.astro.it

2 Astrophysics Group, Blackett Laboratory, Imperial College, Prince Consort Road, SW7 2AZ London, UK

INAF/Osservatorio di Trieste, via Tiepolo 11, 34131 Trieste, Italy

${ }^{4}$ Instituto de Astrofisica de Canarias, C/ via Lactea s/n, 38200 La Laguna, Spain

5 INAF/Osservatorio di Padova, Vicolo Osservatorio 5, 35122 Padova, Italy

Received 14 October 2005 / Accepted 25 January 2006

\section{ABSTRACT}

\begin{abstract}
Aims. We constrain the evolution of the galaxy mass and luminosity functions from the analysis of (public) multi-wavelength data in the Chandra Deep Field South (CDFS) area, obtained from GOODS and other projects, including very deep high-resolution imaging by HST/ACS.

Methods. Our reference catalogue of faint high-redshift galaxies, which we have thoroughly tested for completeness and reliability, comes from a deep $\left(S_{3.6} \geq 1 \mu \mathrm{Jy}\right)$ image by IRAC on the Spitzer Observatory. These imaging data in the field are complemented by extensive optical spectroscopy by the ESO VLT/FORS2 and VIMOS spectrographs, while deep $K$-band VLT/ISAAC imaging is also used to derive further complementary statistical constraints and to assist the source identification and Spectral Energy Distribution (SED) analysis. We selected a highly reliable IRAC $3.6 \mu \mathrm{m}$ sub-sample of 1478 galaxies with $S_{3.6} \geq 10 \mu \mathrm{Jy}, 47 \%$ of which have spectroscopic redshift, while for the remaining objects we used both COMBO-17 data (Wolf et al. 2004, A\&A, 421, 913) and the code Hyperz (Bolzonella et al. 2000, A\&A, 363, 476) to estimate the photometric redshift. This very extensive dataset was exploited to assess evolutionary effects in the galaxy luminosity and stellar mass functions, while luminosity/density evolution is further constrained with the number counts and redshift distributions. The deep ACS imaging allows us to differentiate between these evolutionary paths by morphological type, which our simulations show to be reliable at least up to $z \sim 1.5$ for the two main early- (E/S0) and late-type ( $\mathrm{Sp} / \mathrm{Irr})$ classes.

Results. These data, as well as our direct estimate of the stellar mass function above $M_{*} h^{2}=10^{10} M_{\odot}$ for the spheroidal subclass, consistently show a progressive dearth of such objects starting at $z \sim 0.7$, paralleled by an increase in luminosity. A similar trend, with a more modest decrease in the mass function, is also shared by spiral galaxies, while the irregulars/mergers show an increased incidence at higher $z$. Remarkably, this decrease in the comoving density with redshift of the total population appears to depend on galaxy mass, being stronger for moderate-mass galaxies, but almost absent until $z=1.4$ for high-mass galaxies, thus confirming previous evidence of a "downsizing" effect in galaxy formation.

Conclusions. Our favoured interpretation of the evolutionary trends for the two galaxy categories is that of a progressive morphological transformation (due to gas exhaustion and, likely, merging) from the star-forming to the passively evolving phase, starting at $z \geq 2$ and holding on down to $z \sim 0.7$. The rate of this process appears to depend on galaxy mass, being already largely settled by $z \sim 1$ for the most massive systems.
\end{abstract}

Key words. galaxies: elliptical and lenticular, $\mathrm{cD}$ - galaxies: spiral - galaxies: irregular - infrared: general - infrared: galaxies

\section{Introduction}

As the subject of active, as much as inconclusive, investigation during the last 40 years or so, the cosmological origin of the Hubble galaxy morphological sequence can now be very effectively constrained by combining the unique imaging capabilities of HST/ACS with the wide IR multi-wavelength coverage offered by the Spitzer Space Telescope and the remarkable photon-collecting power and multiplexing of spectrographs on large ground-based telescopes (VLT, Keck). As complex a process as it might have been - involving both gravity and hydrodynamics (see e.g. Baugh et al. 2005), and possibly other physical processes such as black-hole formation and accretion, tidal interactions and merging, and feedback from stellar and nuclear activity (Springel et al. 2005) - we now have a definite chance to observe it in operation.

At the current stage, however, the subject still remains rather controversial. While slow infall of primordial gas may explain disk formation in a relatively simple way, (e.g. Mo et al. 1998), we still lack adequate understanding of spheroid formation. On one side, the homogeneity of the early-type population and tightness of the fundamental plane might suggest that these galaxies have formed from a single monolithic collapse, an early aggregation of lumps of gas turning into stars in the remote past $\left(z_{\text {form }} \geq 3\right.$ ) via a huge burst-like episode followed by quiescence (Eggen et al. 1962; Larson et al. 1975; Chiosi \& Carraro 2002).

This apparently contradicts, however, recently-favoured models of hierarchical galaxy formation postulating that earlytype galaxies are assembled at later times by stochastic 
merging of lower-mass galaxies, either accompanied by strong star-formation (SF) activity (e.g. White et al. 1978; White et al. 1991; Somerville \& Primack 1999; Cole et al. 2000) or through more "silent" dry merging and dynamical aggregation (Bell et al. 2005a; Tran et al. 2005). In such a case, ellipticals would be formed over time scales comparable to the Hubble time, with a major fraction of the mass assembly taking place around $z \sim 1$ (e.g. Somerville et al. 1999), and virtually all massive galaxies disappearing by $z \geq 1.5$. Benson et al. (2002) find that popular hierarchical models produce as many spheroids with highly inhomogeneous colours as are observed locally, but that they underpredict the proportion of homogeneous, passive objects at redshifts $z \sim 1$. This suggests that, while the star-formation rate (SFR) in spheroidals at low redshifts $(z \leq 1)$ is predicted correctly, the formation rate at higher redshifts is underestimated. On the other hand, recent results from the K20 project (see Daddi et al. 2004a, and references therein) claim that semianalytic models underestimate the number of massive galaxies at $z \sim 2$ by about a factor of 30 and suggest that the assembly of massive galaxies took place at substantially earlier epochs than predicted by these models.

Observational constraints on the SF history have been inferred from the broad-band colours, line-strength indices and stellar chemical abundances. When referred to massive ellipticals, these observations often suggest that the bulk of stars might have been formed in a remote past. However, some secondary activity of SF in the recent past is also evident: nearby ellipticals both show a wide variety of morphological and kinematical peculiarities (e.g. Longhetti et al. 2000) and a considerable spread in stellar ages, particularly for the field population (Thomas et al. 2005). Strong evolution in the population of early-type galaxies has been reported by Kauffmann et al. (1996) and Kauffmann \& Charlot (1998), which has been considered to support the hierarchical galaxy-formation models.

Published results from high-redshift galaxy surveys appear not infrequently in disagreement with each other, and conflicting conclusions are reported (see Faber et al. 2005 for a recent review about galaxy evolution at $z<1$ ). This is partly due to the small sampled areas and the corresponding substantial fieldto-field variance. However, a more general problem stems from the apparent conflict between the detections of massive galaxies at very high redshifts (e.g. Cimatti et al. 2004; Glazebrook et al. 2004; Labbé et al. 2005; Daddi et al. 2005a,b) and indications of a fast decline in the comoving number density at $z>1$ (Franceschini et al. 1998; Fontana et al. 2004).

In summary, while early monolithic collapse and hierarchical models imply radically different histories for spheroids, neither the theoretical predictions nor the observational constraints for field galaxies have been sufficiently definitive as yet for precise conclusions to be drawn in favour of one or the other scenario.

The most direct way of constraining the evolutionary history of galaxies and trying to resolve the discrepancies would be to derive the redshift-dependent luminosity and mass functions from deep, unbiassed surveys. This has been pursued by a number of teams, relying on either $U$-band optical selection as a probe of SFR density (Lilly et al. 1996; Madau et al. 1996) and UV selection by GALEX (Schiminovich et al. 2005) or on observations in the $K$-band (Cowie et al. 1996; Cimatti et al. 2002; Dickinson et al. 2003; Franx et al. 2003; Drory et al. 2004; Fontana et al. 2004; Bundy et al. 2005).

Here we contribute to this effort by exploiting very deep public imaging by the IRAC photometric camera on the Spitzer Observatory to select a more unbiassed sample of high- $z(z \leq 2)$ near-IR galaxies. We used for this the IRAC Channel-1 $3.6 \mu \mathrm{m}$ data over $160 \operatorname{arcmin}^{2}$ in the Chandra Deep Field South (CDFS) taken within the GOODS project (Dickinson et al. 2004). The other IRAC imaging data in the field are either redundant (Channel-2 at $4.5 \mu \mathrm{m}$, too close to channel-1 and somewhat less sensitive) or include non-stellar contributions by the galaxy ISM (the longer wavelength Channels-3 and 4) that would furtherly complicate the interpretation.

Near-IR surveys are best suited to the study of faint highredshift galaxy populations for various reasons. Compared to UV-optical selection, the observed fluxes are minimally affected by dust extinction. At the same time, they are good indicators of the stellar mass content of galaxies (Dickinson et al. 2003; Berta et al. 2004), and closer to providing a mass-selection tool. For typical spectra of evolved galaxies, the IRAC Channel-1 $3.6 \mu \mathrm{m}$ also benefits from a K-correction that is particularly favourable to the detection of high-redshift galaxies, particularly if we consider that the $H^{-}$opacity minimum (corresponding to a typical peak in galaxy's SEDs) at $\lambda \simeq 1.7 \mu$ m in stellar atmospheres (Simpson \& Eisenhardt 1999) falls within the waveband of Channel-1 for $z=1$ objects.

In addition to the Spitzer observations, the GOODS project has provided the community with an unprecedented amount of high-quality optical and near-IR data in CDFS, particularly the very deep 4-band ACS imaging, thereby allowing the most accurate morphological analysis currently possible over an appreciable area. Building on our previous experience of faintgalaxy imaging and statistical modelling (Rodighiero et al. 2001; Cassata et al. 2005), on our tools for spectro-photometric analysis (Poggianti et al. 2001; Berta et al. 2004), and on reduction of deep IR imaging data from space (e.g. Rodighiero et al. 2004 for ISO data; Lonsdale et al. 2004; Hatziminaoglou et al. 2005; Rodighiero et al. 2005 for Spitzer data), we illustrate in this paper the power of combining such multi-wavelength information for analysing the evolutionary mass and luminosity functions of faint galaxies.

Since the spectroscopic follow-up is currently only partial in the field, and to avoid confusion problems in the IRAC data as far as possible, we limit our analysis to moderate depths. In spite of this, the constraints on the history of massive galaxy evolution are already relevant. Pushing the analysis to the IRAC sensitivity limits will allow us to extend our conclusions further down in mass/luminosity and up in $z$.

Though substantially larger than HDF's, our survey field still has a moderate size. Eventually, a complete understanding of the influence of large-scale structures on the evolutionary history and to reduce the effects of cosmic variance will need newgeneration datasets on large areas (e.g. COSMOS, Scoville et al. 2004).

The paper is structured as follows. Section 2 describes the optical and IR (Spitzer) data, the near-IR data and ACS/HST imaging, the spectroscopic data used in our analysis, and our criteria for catalogue combination and merging. Section 3 gives details of our quantitative morphological analysis of faint galaxies, and Sect. 4 summarises our effort for the photometric redshift estimate. Our statistical analyses are reported in Sect. 5, while Sect. 6 is dedicated to deriving the mass function in the stars of our galaxies. A comparison of observed and model number counts is discussed in Sect. 7. Sections 8 and 9 summarise our results and conclusions.

We adopt in the following a standard set of values for the cosmological parameters $\Omega_{\mathrm{M}}=0.3$ and $\Omega_{\Lambda}=0.7$, while for ease of comparison with other published results, we express the dependence on the Hubble constant in terms of the parameter 
$h \equiv H_{0} / 100 \mathrm{~km} \mathrm{~s}^{-1} \mathrm{Mpc}^{-1}$ and provide the relevant scaling factors.

\section{Observations and data analysis}

\subsection{Deep IR imaging with Spitzer}

The GOODS southern field is located in the Chandra Deep Field South $(\mathrm{CDFS})$ area, that is centred at RA $(\mathrm{J} 2000)=03: 32: 30.37$ and $\operatorname{Dec}(\mathrm{J} 2000)=-27: 48: 16.8$. The IRAC Spitzer observations in the field include deep imaging in four near-IR bands $(3.6,4.5$, 5.8 , and $8.0 \mu \mathrm{m})$. The exposure time per channel per sky pointing was $23 \mathrm{~h}$ as a minimum. Due to the fact that observations were done in two epochs, it is approximately two times this in a central deeper strip where the two overlap. In this paper we exploit a galaxy catalogue that we have derived from the $3.6 \mu \mathrm{m}$ IRAC data.

We started the data reduction using products generated by the Spitzer Science Center (SSC) Basic Calibrated Data (BCD) pipeline. We used all the BCDs available in the archive at the end of November 2004. The archived data were processed with pipeline version S10.5.0 provided by the Spitzer Science Center. We applied an additive correction factor to each BCD frame in order to remove the median background. We have processed and mosaiced all corrected BCDs within the Mopex package ${ }^{1}$, using a standard procedure that accounts for cosmic radiation hits, outliers, flat-field, and distortions of the detector. The pixel size in the final map is 0.6 arcsec/pixel. The GOODS IRAC mosaic with a significant sky coverage (sky pixel repetition factor $>20$ ) covers an area of approximately $12 \times 18 \mathrm{arcmin}^{2}$ on the sky.

The IRAC source extraction was performed with SExtractor (Bertin \& Arnouts 1996). Assuming that essentially all the sample sources are seen as point-like by the IRAC $\sim 3$ arcsec imager, we computed the fluxes within a 5.9 arcsec diameter aperture and applied a correction factor derived from the stars in the IRAC images to assess the total fluxes. In the case of extended sources, we used Kron like magnitudes (AUTO_MAG output parameter in SExtractor).

\subsection{Near-IR ground-based imaging}

As part of GOODS, near-infrared imaging observations of the CDFS were carried out in $J, H, K_{\mathrm{s}}$ bands, using the ISAAC instrument mounted on the ESO VLT telescope. We made use of the publicly available $J$ and $K$ band imaging (version 1.0, released $^{2}$ by the ESO/GOODS team in April 2004). This data release includes 21 fully reduced VLT/ISAAC fields in $J$ and $K_{\mathrm{s}}$ bands, covering $130 \operatorname{arcmin}^{2}$ of the GOODS/CDFS region. It also includes mosaics of the co-added tiles as single FITS files in $J$ and $K_{\mathrm{s}}$ bands, as well as the corresponding weight-maps.

To provide a homogeneous photometric calibration across the entire field, the GOODS team at ESO have rescaled all images to the same zero point (26.0 in the AB system). The final mosaics have a pixel scale of $0.15^{\prime \prime}$. We ran SExtractor on the $J$ and $K$ mosaics to obtain total magnitudes (BEST_MAG output parameter in SExtractor) for all the objects in the field.

\footnotetext{
${ }^{1}$ Mopex performs the processing and mosaicing of both IRAC and MIPS Spitzer imaging data. Details can be found in http://ssc.spitzer. caltech.edu/postbcd/.

2 http://www. eso.org/science/goods/releases/20040430/.
}

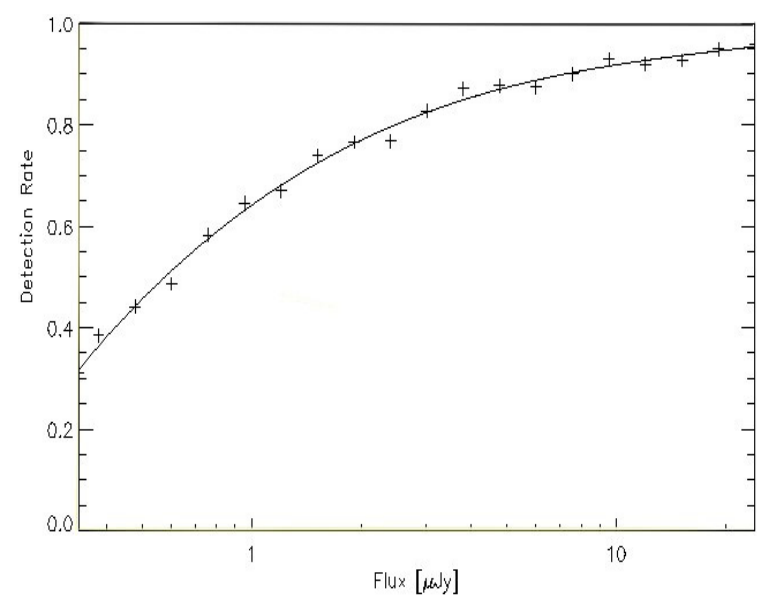

Fig. 1. Selection function of the $3.6 \mu \mathrm{m}$ band Spitzer sample in the CDFS, as from our Monte Carlo simulations. See text for details.

\subsection{The near-IR source selection functions}

The completeness of our $3.6 \mu \mathrm{m}$ catalogue was assessed through numerical simulations. A number of sources spanning the $0.3-30 \mu \mathrm{Jy}$ flux range were added to the image at random positions using a synthetic TinyTim PSF (Krist 2002). A conservative overall figure of 1600 sources (corresponding to 200 beams/source) was chosen as the additional source density in order to avoid confusion effects.

Source extraction was carried out as on the original image, and inputs and outputs were cross-correlated using a 1.5 pixel search radius (corresponding to 0.9 arcsec). The results are shown in Fig. 1, where a fit to the selection function curve is also shown. Our sample turns out to be $\sim 60 \%$ complete above $1 \mu \mathrm{Jy}, \sim 90 \%$ complete above $5 \mu \mathrm{Jy}$, and more than $\sim 95 \%$ above $10 \mu \mathrm{Jy}$. Further tests of the validity of our IRAC source selection will be discussed in Sect. 5.1 based on a comparison of our own and independent results on the galaxy number counts. The IRAC $3.6 \mu \mathrm{m}$ sample at 1 (10) $\mu \mathrm{Jy}$ includes 5622 (1646) sources, respectively.

We also performed extensive Monte Carlo simulations, including inserting several IRAC/GOODS-like toy galaxies in the real image, to determine the limit of completeness in the $K$ band. We applied the same extraction criteria used on the ISAAC images and derived the detection rate as a function of the simulated magnitude. We estimated the $K$-band sample to be more than $90 \%$ complete at $K<21$ (Vega reference system).

\subsection{ACS/HST optical imaging}

The core of the GOODS project was the acquisition and reduction of high-resolution HST/ACS imaging data obtained as an HST Treasury Program (Giavalisco et al. 2004). The GOODS ACS/HST Treasury Program has surveyed two separate fields (the CDFS and the Hubble Deep Field North) with four broadband filters: $F 435 W(B), F 606 W(V), F 775 W(i)$, and $F 850 L P(z)$. Observations in the $V, i$, and $z$ filters were split into five epochs, separated by about 45 degrees, in order to detect transient objects. Observations in the $B$ band are taken during epoch 1 for both fields. Images taken at consecutive epochs have position angles increasing by $45^{\circ}$. The total exposure times are $2.5,2.5$, 5 orbits in the $V, i$, and $z$ bands, respectively. The exposure time in the $B$ band is three orbits. In August 2003 the GOODS team released version 1.0 of the reduced, stacked, and mosaiced images for all the data acquired over the five epochs of observation. 
To improve the point spread function (PSF) sampling, the original images, which had a scale of 0.05 arcsec pixel $^{-1}$, have been drizzled on to images with a scale of $0.03 \operatorname{arcsec}_{\text {pixel }}{ }^{-1}$. We used the version 1.0 of the image catalogue.

The data set is complemented with the ACS/HST catalogs released by the HST/GOODS team in October 2004, containing the photometry in $B, V, i$, and $z$ bands. The source extraction and the photometric measurements have been performed by the GOODS team running a modified version of SExtractor on the version 1.0 images. Particular attention was payed to the photometry of faint sources, thanks to careful determination of the local sky background. As for the case of the $K$-band imaging, we have considered the total magnitudes (as from the BEST_MAG output parameter in SExtractor). Moreover, for purposes of a detailed morphological analysis, we used the high-resolution deep imaging carried out by ACS/HST in the $z$-band (version 1.0, Giavalisco et al. 2004).

\subsection{Optical spectroscopy}

In the past few years ESO has performed various systematic spectroscopic observational programs in the CDFS area by using all available multi-object spectrographs (i.e. FORS1, FORS2, VIMOS; see Cimatti et al. 2002b; Le Fevre et al. 2004a,b; Szokoly et al. 2004; Vanzella et al. 2005). The contributions of the major projects are hereby summarised.

The VIMOS VLT Deep Survey (VVDS, Le Fevre et al. 2004a) observed a large sample of galaxies in the CDFS area. The redshift data have been released to the community ${ }^{3}$ : a total of 1599 objects with $I_{\mathrm{AB}} \leq 24$ have measured redshifts. The completeness in redshift measurement for the targeted objects is high, better than $84 \%$, and 784 of these fall within the GOODSSouth ACS field.

In the framework of the GOODS project, a large sample of galaxies in the CDFS has been spectroscopically targeted (Vanzella et al. 2005). A total of 303 objects with $z_{850} \lesssim 25.5$ were observed with the FORS2 spectrograph, providing 234 redshift determinations. The reduced spectra and the derived redshifts are released to the community ${ }^{4}$.

Further GOODS spectroscopic observations have been carried out with FORS2 at VLT (Szeifert et al. 1998) using the 300I grism, without blocking filter (ESO programs 170.A-0788 and 074.A-0709). This configuration gives a low resolution $(R \sim$ $850)$ in the wavelength range $\sim 5600-10000 \AA$. Data reduction is explained in Vanzella et al. (2005).

Another fraction of the spectroscopic redshifts were obtained as part of the K20 survey (Cimatti et al. 2002b), which have also been recently made public ${ }^{5}$. The K20 sample includes 546 objects to $K_{\mathrm{s}} \leq 20$ (Vega system) over two independent fields (52 $\operatorname{arcmin}^{2}$ in total, $32 \operatorname{arcmin}^{2}$ in the CDFS). The spectroscopic redshift completeness is $94 \%$ and $87 \%$ for $K_{\mathrm{s}} \leq 19$ and $K_{\mathrm{s}} \leq 20$, respectively.

All the spectroscopic redshifts available in version 1.0 of the GOODS/CDFS ACS catalogue have been compiled in a master list $^{6}$.

\footnotetext{
${ }^{3}$ http://cencosw.oamp. fr/.

4 http://www.eso.org/science/goods/.

5 http://www.arcetri.astro.it/ k20/ spe_release_dec04/index.html/.

${ }^{6}$ http://www.eso.org/science/goods/ spectroscopy/CDFS_Mastercat/.
}

\subsection{Infrared to optical source associations}

The excellent quality of HST imaging allowed us to obtain reliable associations for most of the Spitzer IR sources with the corresponding optical counterparts. On the other hand, the much worse spatial resolution of IRAC compared to ACS implies some significant complication in source association, which is discussed below.

It should be noticed that the deep $K$-band ISAAC images should provide decisive support for the identification. However, only a fraction $(\sim 60 \%$, see Sect. 2.6.4) of the IRAC Spitzer $3.6 \mu \mathrm{m}$ sources turned out to have counterparts in the $K$-band (partly because the ISAAC imaging covered only $\sim 80 \%$ of the Spitzer/ACS common area). For this reason, our identification of the Spitzer sources followed a two-step procedure.

We first looked for optical identifications by matching with the ACS $z$-band catalogue. This provided us with reliable associations for a large majority of the $3.6 \mu \mathrm{m}$ objects. We sthen proceeded to match all Spitzer sources with sources extracted from the ISAAC $K$-band images. This second step was needed not only to obtain near-IR data essential for the SED fitting, photometric redshifts, etc., but also to disentangle the correct identification for dubious matches, multiple associations, or confused sources emerging from step one.

\subsubsection{Associations with the ACS $z$-band data}

The validity of the association of the $3.6 \mu \mathrm{m}$ with $z$-band ACS sources was verified here by using the likelihood ratio technique introduced by Sutherland \& Saunders (1992). We adopt here the formalism described by Ciliegi et al. (2003).

The likelihood ratio $(L R)$ is defined as the ratio between the probability that a given source at the observed position and with the measured magnitude is the true optical counterpart, and the probability that the same source is a chance, unrelated object:

$L R=q(m) f(r) / n(m)$

where $n(m)$ is the surface density of objects with magnitude $m$, $q(m)$ the expected distribution as a function of magnitude of the optical counterpart, and $f(r)$ the probability distribution function of the positional errors.

In the presence of more than one counterpart in the error box, the reliability $\operatorname{Rel}_{j}$ for object $j$ being the correct identification is:

$\operatorname{Rel}_{j}=\frac{(L R)_{j}}{\sum_{i}(L R)_{i}+(1-Q)}$

where the sum is over the set of all candidates for this particular source and $Q$ is the probability that the optical counterpart of the source is brighter than the magnitude limit of the optical catalogue $\left(Q=\int^{m_{\lim }} q(m) \mathrm{d} m\right)$.

For each IRAC source we adopted a mean positional error of $1 \mathrm{arcsec}$, and we assumed a value of $0.1 \mathrm{arcsec}$ as the optical positional uncertainty. We chose a search radius of 1.5 arcsec from the position of the centroid of the infrared error box to look for the possible optical counterparts. Figure 2 shows the resulting magnitude distribution of the correct identifications together with the expected distribution of background objects unrelated to the infrared sources. The smooth curve fitted to the former has been used as input in the likelihood calculation. Since the fraction of infrared sources with more than one possible optical counterpart is $\sim 15 \%$ (see below), this corresponds to an expected fraction of correct identifications above the magnitude limit of the optical catalogue on the order of $\sim 85 \%$. On this basis we 


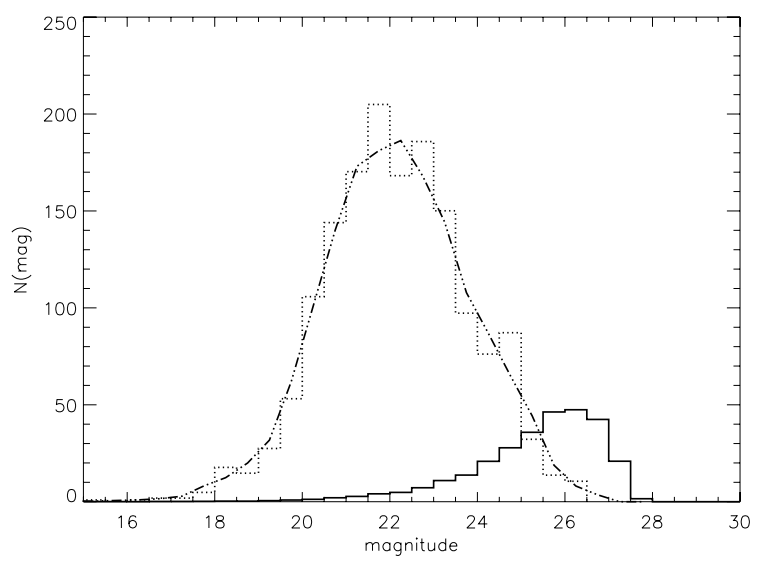

Fig. 2. AB magnitude distributions of background objects (solid line) and "real" detections (real $(m)$, dotted line) estimated from the optical objects detected in the $z$ band within a radius of 1.5 arcsec around each infrared sources. The smooth curve fitted to the real $(\mathrm{m})$ distribution (dot dot dot dashed line) has been used as input in the likelihood calculation.

adopted for the $Q$ parameter the value $Q=0.85$. To check how this assumption could affect our results, we repeated the analysis using different $Q$ values in the range 0.5-1.0: no substantial difference in the final number of identifications and in the associated reliability has been found.

Once $q(m), f(r)$, and $n(m)$ were determined, we computed the $L R$ value for all the optical sources within a distance of 1.5 arcsec from the infrared positions. The best threshold value for $L R\left(L_{\mathrm{th}}\right)$ then needed to be defined, in order to discriminate between spurious and real identifications. As discussed by Ciliegi et al. (2003), $L_{\text {th }}$ has to be small enough to avoid missing many real identifications, and be large enough to keep the number of spurious identifications as low as possible. For the $L R$ threshold we adopted the value $L_{\mathrm{th}}=0.15$. With this and from Eq. (2) with $Q=0.85$, all the optical counterparts of infrared sources with only one identification (the majority in our sample) and $L R>L R_{\text {th }}$ have a reliability greater than 0.5 . The $L R$ analysis has been performed for the whole IRAC sample to $S_{3.6}=1 \mu \mathrm{Jy}$.

In order to minimize the problem of misidentification, we checked by visual inspection all the optical associations of each IR source with $S_{3.6 \mu \mathrm{m}}>10 \mu \mathrm{Jy}$, our highly reliable and complete sub-sample. For stars and isolated objects, the crosscorrelation is unambiguous: the shape and the peak of the infrared contours overlayed on the $z$ image have confirmed the associations. However, for several IRAC sources, particularly for the extended ones, the relationship between infrared and optical emissions may be much more uncertain: few optical sources can lie inside the IRAC error box and contribute to the infrared emission. Such cases are dealt with in the following subsections.

\subsubsection{Multiple associations and confused sources}

We found that $\sim 10 \%(\sim 15 \%)$ of the $S_{3.6 \mu \mathrm{m}}>10 \mu \mathrm{Jy}$ sources have more than one optical counterpart within 1 (1.5) arcsec from the IR centroid position. Examples are shown in Fig. 3, where we report zooms on the optical $z$-band image with the $3.6 \mu \mathrm{m}$ contours overlaid (red lines) for a couple of sources. The black squares mark the positions of the original IRAC catalogue, while the blue circles are the optical counterparts. The thicker blue circles indicate our final associations, chosen by the automatic procedure discussed above.
In many such cases, the $L R$ analysis was able to disentangle the correct identification: in the lack of independent information (Sect. 2.6.3) we assumed that the object with the highest likelihood ratio value was the correct counterpart of the IRAC source.

A fraction of the IR sources with multiple optical counterparts display clear signs of confusion, due to the largely different spatial resolution of the Spitzer and HST images. In such cases an extended $3.6 \mu \mathrm{m}$ emission appears to emerge from more than one optical source (generally by no more than two), and there is no obvious indication of the level of contribution by the various optical components to the IR flux. An example is given in Fig. 4: in this case the two brighter optical sources are at the same redshift $(z \sim 0.37)$. The fraction of confused sources in our matched IRAC/ACS sample above $S_{3.6 \mu \mathrm{m}}=10 \mu \mathrm{Jy}$ is on the order of $\sim 5 \%$.

Concerning the part of the sample with fluxes between 1 and $10 \mu \mathrm{Jy}$, the fractions of sources with multiple optical associations (hence with potential confusion problems) is $16 \%$ for an association radius of 1 arcsec and $28 \%$ for 1.5 arcsec radius. For these we used the $L R$ value to identify the correct optical counterpart.

\subsubsection{Matching with the ISAAC K-band imaging}

To reduce the level of contamination and confusion in our samples, we made full use of the deep ISAAC $K$-band imaging, which is close enough to the $3.6 \mu \mathrm{m}$ selection wavelength and at the same time provides sub-arcsec resolution.

We cross-correlated the $z$-band ACS catalogue with the ISAAC image in the $130 \mathrm{arcmin}^{2}$ common area, by applying the $L R$ analysis presented in Sect. 2.6.1. This comparison allowed us to resolve the majority of the confusion problems in the complete $10 \mu \mathrm{Jy}$ sample. Our procedure was to split the $3.6 \mu \mathrm{m}$ confused source into as many components as revealed by the ISAAC image (typically two), and to assign a flux to each one according to the observed ratios of the fluxes in the $K$-band. An example of the application of this procedure is reported in Fig. 4. In such a way we have resolved $60 \%$ of the confused cases, and brought the fraction of confused sources to only $2 \%$ of the complete sample.

Due to the small number and negligible impact on our results of the latter, we have not attempted to apply complex deblending procedures to recover the $3.6 \mu \mathrm{m}$ flux coming from each single component. Instead, when building the observed SEDs for these 30 objects, we summed the optical fluxes of the components of the IRAC blend, at least where there was evidence of interaction from morphology (see the example in Fig. 4).

In conclusion, our choice of a moderate flux limit $\left(S_{3.6 \mu \mathrm{m}}=\right.$ $10 \mu \mathrm{Jy}$ ) for our reference complete sample minimizes the photometric complications due to source confusion. For the fainter sample, used in the following only for extracting morphologyselected number counts, application of the procedure of using the ISAAC image did not bring a significant improvement in the statistics of multiple or confused sources.

\subsubsection{The band-merged IRAC/GOODS catalogue}

Once each $3.6 \mu \mathrm{m}$ source was assigned to its $z$-band - and whenever possible $K$-band - counterparts, we proceeded by building a multi-wavelength catalogue, in order to include all the available photometric bands. The cross-correlation with the ISAAC $(J$ and $K$ ) and the other HST bands was done using a search radius of 1.5 arcsec around the $z$-band positions. The area covered by HST observations, approximately $\sim 160 \mathrm{arcmin}^{2}$, is smaller than 

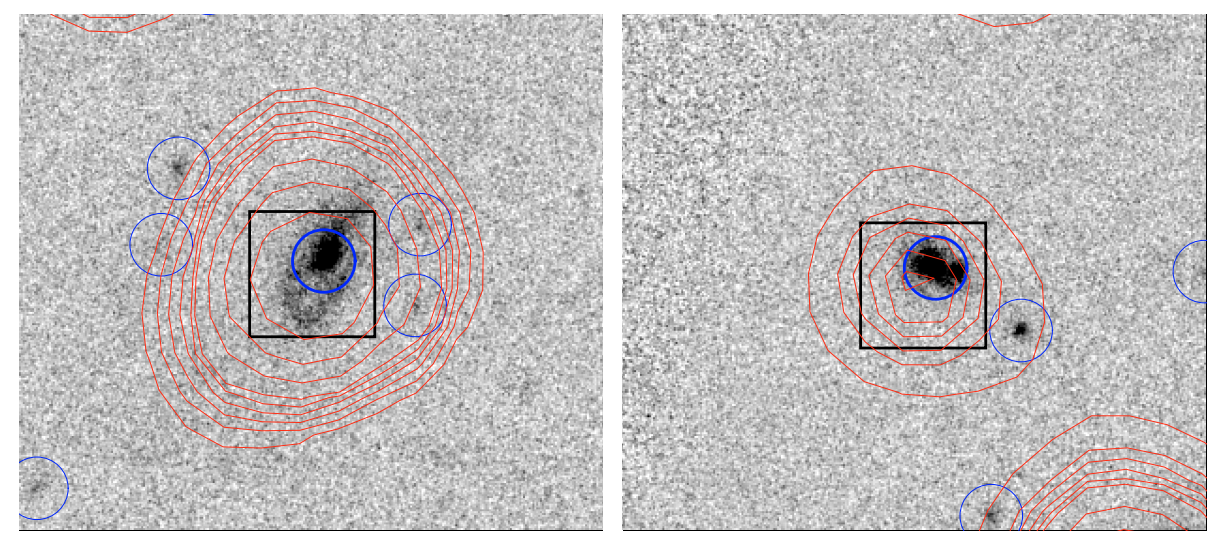

Fig. 3. Examples of IRAC sources with multiple optical counterparts. For the two sources the $3.6 \mu$ m contours (red lines) are overlaid on a zoom of the optical $z$-band ACS image $\left(9^{\prime \prime} \times 8^{\prime \prime}\right.$ size). The black squares mark the positions of the original IRAC centroids, while the blue circles denote the optical counterparts. The thicker blue circles indicate our final associations, chosen with the automatic procedure discussed in Sect. 2.6.
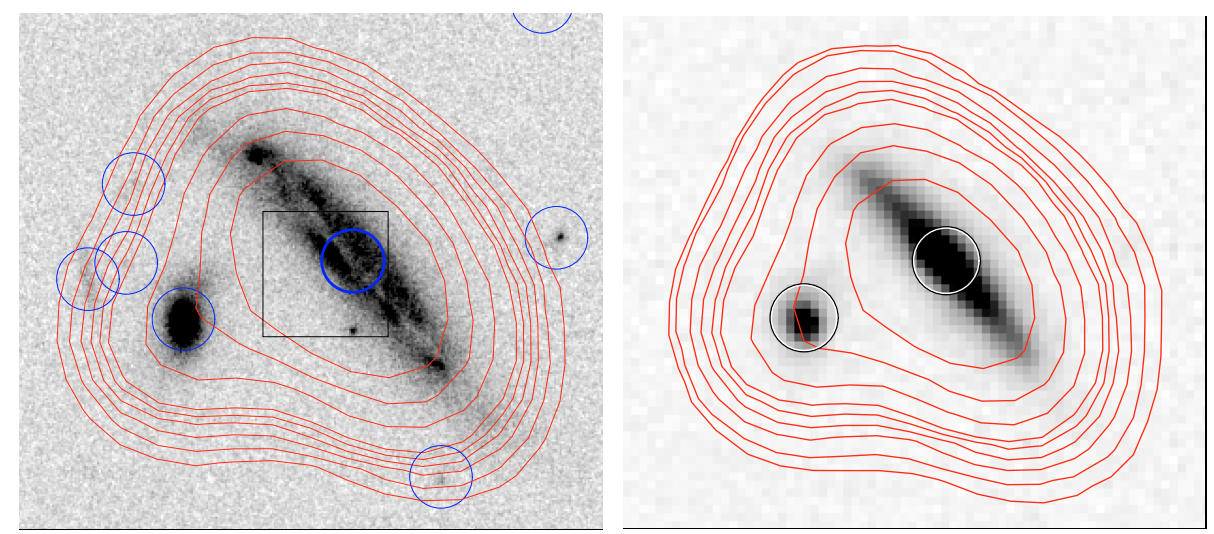

Fig. 4. Example of a blended IRAC source. The meaning of the line types and the image size are the same as in Fig. 3. The left panel reports an overlay of the IRAC $3.6 \mu \mathrm{m}$ contours over the $z$-band ACS image. The two brighter optical sources, contributing to the IRAC flux, are at the same redshift $(z \sim 0.37)$. The right hand panel overlays the IRAC contours to the ISAAC $K$-band image. The two components take $83 \%$ and $17 \%$ of the $K$ flux, corresponding to $K_{\mathrm{AB}}=18.72$ and 20.50. This is adopted here as the flux ratio of the two sources assumed to produce the $3.6 \mu \mathrm{m}$ blend.

that originally covered by Spitzer, $81 \%$ of which $\left(130 \operatorname{arcmin}^{2}\right)$ is also surveyed in $K$ by ISAAC.

Our final sample includes 1646 IRAC sources with $S_{3.6 \mu \mathrm{m}}>$ $10 \mu \mathrm{Jy}$ (1478 turn out to be galaxies and 168 stars, see below) and a total of 5622 sources with $S_{3.6 \mu \mathrm{m}}>1 \mu \mathrm{Jy}$ inside the Spitzer/ACS common area of $160 \mathrm{arcmin}^{2}, 5302$ of which are galaxies and 320 stars. Sixty percent of the total sample sources have a $K$-band counterpart. This fraction reaches $75 \%$ when considering the high-reliability limit of $S_{3.6 \mu \mathrm{m}}>10 \mu \mathrm{Jy}$.

\section{Morphological classification}

We performed a quantitative morphological classification using the $z$-band ACS images for all sample galaxies. For this we measured for each galaxy the set of parameters Concentration $(C)$, Asymmetry $(A)$, and clumpinesS $(S)$ of the galaxy light distribution (CAS parameters).

The concentration roughly correlates with the bulge-to-disk ratio, allowing distinction between bulge- and disk- dominated galaxies (Abraham et al. 1996). The asymmetry distinguishes normal isolated galaxies from either irregular/merging systems (Conselice et al. 2000). The clumpiness (a measure of the uniformity of the galaxy surface brightness distribution) is expected to correlate with the ongoing SF rate (Conselice 2003a).

When attempting a morphological classification of a large sample of galaxies at widely different redshifts, it is crucial to take the effects of the so-called morphological K-correction into account. This considers that galaxies observed at bluer wavelengths tend to display a later morphological type. For this reason we have used the longest wavelength $z$-band (the F850LP ACS filter) to study the morphology of the entire sample: this band corresponds to about rest-frame $I, V, B$, and $U$ for galaxies at $z=0.2,0.5,1$, and 1.5 , respectively.

However, as already discussed by various authors (Windhorst et al. 2002; Papovich et al. 2003), this effect is relevant mainly when comparing $U$ rest-frame with visual ( $B$ or $V$ rest-frame) morphologies. This means in our case that only some of the galaxies at a redshift larger than $z \sim 1.5$ may be significantly influenced by this effect. Cassata et al. (2005, henceforth CA05) discuss in detail and try to quantify it by comparing the visual morphology of a sample of $z \sim 1$ galaxies in their $U$ and $B$ rest-frame. They find that the morphology is preserved for all early-type galaxies, while some $B$ rest-frame disks appear irregular in the $U$ rest-frame (but this is not important in our approach, which segregates only early- from late-type galaxies). Given that the technique used for this work is calibrated on the results by Cassata et al. (2005), the same conclusions of that work can be applied here. Therefore, we do not expect our analysis to be affected by strong morphological $\mathrm{K}$-correction effects, especially considering that our main points about the evolutionary mass and luminosity functions in the following sections. concern the $z<1.5$ universe, and since we 


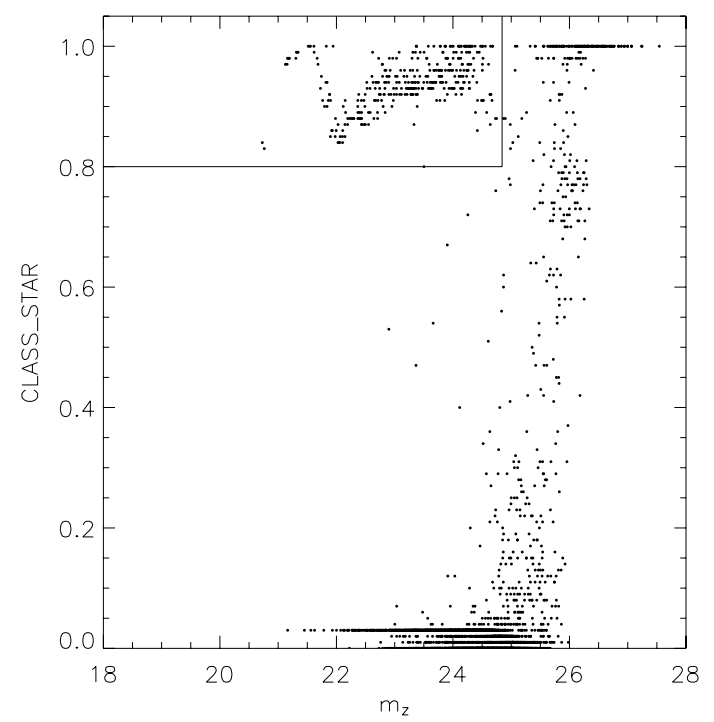

Fig. 5. Plot of the stellarity index $\left(C L A S S \_S T A R\right)$ from the SExtractor analysis versus the $z_{\mathrm{AB}}$ magnitude. Stars are selected in the rectangular box of high stellarity and bright magnitude.

confine our analysis to the basic early- to late-type structural differentiation.

Morphological classification based on the CAS parameter set is particularly effective in disentangling spheroid-dominated from spiral/irregular galaxies. Early type galaxies usually have a low value for asymmetry, high concentration and small clumpiness. Cassata et al. (2005) find that early type galaxies in the K20 catalogue (a subset of the current GOODS sample, Cimatti et al. 2002) occupy a rather precise domain of the CAS parameter space, as measured on the ACS/GOODS images:

$$
A<0.2 ; \quad C>2.7 \quad 0.0<S<0.3 ; \quad 3 A-S<0.3 \text {. }
$$

Galaxies having CAS parameters lying in the region above are classified as early-type, while the remaining are classified as late-type. The stars are very effectively isolated by combining the $z$-band flux with the stellarity index calculated by SExtractor (CLASS_STAR) on the $z$-band images. Figure 5 plots the stellarity index $\left(C L A S S \_S T A R\right)$ versus the $z_{\mathrm{AB}}$ magnitude. Stars are easily identified in the rectangular box of high stellarity and bright magnitude.

We have first applied the above technique to the 1646 objects of our $3.6 \mu \mathrm{m}$ catalogue above $10 \mu \mathrm{Jy}$, and identified in this 168 stars, 472 (32\%) early-type and 1006 (68\%) late-type galaxies in the complete sample. This compares with a fraction of $26 \%$ early-types (74\% spirals and irregulars) estimated by Bundy et al. (2005) among galaxies brighter than $z_{\mathrm{AB}}=22.5$.

The morphology of a subsample of 155 galaxies (about $10 \%$ of the objects in our $10 \mu \mathrm{Jy}$ catalogue) has also been visually inspected, in order to check the reliability of our automatic technique. We found that the visual and the CAS morphologies agree with each other for $90 \%$ of the 55 galaxies automatically classified as early-type and for $97 \%$ of those automatically classified as late-types. The automatic classification procedure then provides a valuable and robust tool for disentangling early from late morphological type galaxies.

We have then addressed the more challenging problem of estimating structural properties for sources fainter than $10 \mu \mathrm{Jy}$ and down to $S_{3.6}=1 \mu \mathrm{Jy}$. To this end, we have applied our method to all 3976 sources detected within this flux interval, of which 152 turned out to be stars, 548 early-type, and 3276 latetype galaxies. Then the fraction of spheroidal galaxies to total drops from more than $30 \%$ in the bright sample to $14 \%$ fainter than $S_{3.6}=10 \mu \mathrm{Jy}$.

It should be noted that, among all objects in this flux interval, 990 have $z$-band ACS magnitude fainter than 25 (927 of which classified by CAS as late-type, 61 as early-type and 2 stars). Our simulation experiments (see also Cassata et al. 2005) indicate that, beyond $z_{\mathrm{AB}} \simeq 25 \mathrm{mag}$, the automatic classification is less reliable.

For this reason we performed a visual check of a large subset of the IRAC/GOODS galaxy sample, with particular attention to the 990 optically fainter, in random-magnitude order, to determine how many would be consistent with an earlytype and how many with a late-type morphology. As for the 548 CAS-classified ellipticals, visual inspection shows that approximately $30 \%$ may be consistent with having misclassified late-type morphologies. On the other hand, a CAS-classified late-type galaxy has a much lower chance (on the order of $4 \%$ at the $1 \mu \mathrm{Jy}$ limit) to be a spheroidal system.

In conclusion, for spheroidal galaxies we calculate that the overall uncertainty due to morphological misclassification of the faint optical counterparts is such that their number ranges from 380 to 690 objects (i.e. from $10 \%$ to $18 \%$ of the total galaxy population) at the $1 \mu \mathrm{Jy}$ sensitivity limit of our survey. The corresponding relative uncertainty in the late-type morphological subset is lower by a factor $\sim 5$, or on the order of $20 \%$ at most. This provides us with the upper and lower boundaries in the number counts differentiated by morphological class (see Sect. 5.1).

\section{Photometric redshift}

The spectroscopic data available in the GOODS/IRAC/GOODS area (see Sect. 2.5) were cross-correlated with our $S_{3.6 \mu \mathrm{m}}>$ $10 \mu \mathrm{Jy}$ complete catalogue, including 1478 extragalactic sources at this flux limit. Of these, 695 (or $47 \%$ ) turned out to have a spectroscopically confirmed counterpart.

For a large fraction of the remaining galaxies, we used public photometric redshifts from COMBO-17 (Wolf et al. 2004), a multi-band photometric survey entirely covering our field. Wolf et al. show that the COMBO-17 photometric redshifts are highly reliable and accurate for galaxies with $R<24$ and $z<1$, for which the typical $1-\sigma$ redshift error $\sigma_{z} /(1+z)$ is $\sim 0.07$. The comparison of the photometric redshift estimate and spectroscopic measurements shows, however, an increasing scatter from the one-to-one relation above $z_{\text {phot }}>0.8$ (see Fig. 4 in Wolf et al. 2004). This observed discrepancy between the photometric and the spectroscopic data at high $z$ is mostly due to the fact that COMBO-17 is only based on optical SEDs, and cannot exploit the Balmer-jump spectral feature for galaxies at $z>1$. Altogether, of our 783 galaxies without spectroscopic redshift, we have used 375 photometric estimates from COMBO-17, all those with $z_{\text {phot }} \leq 0.8$. One hundred and 26 IRAC $3.6 \mu \mathrm{m}$ sources do not have a COMBO-17 association due to the different selection functions.

For the remaining 408 sources $(27 \%)$ in our sample, photometric redshifts were estimated here using the Hyperz code (Bolzonella et al. 2000), exploiting the availability of an extensive photometric coverage at $\lambda>1 \mu \mathrm{m}$. In particular we included the IRAC $3.6 \mu \mathrm{m}$ fluxes in our analysis, as providing a useful constraint on the solutions (e.g. Rowan-Robinson et al. 2005; Polletta et al. in prep.). While fine-tuning the procedure, it turned out that fairly accurate photometric redshifts were obtained by using only two templates from the set provided by Hyperz, namely one for ellipticals (E.ised) and one for normal 

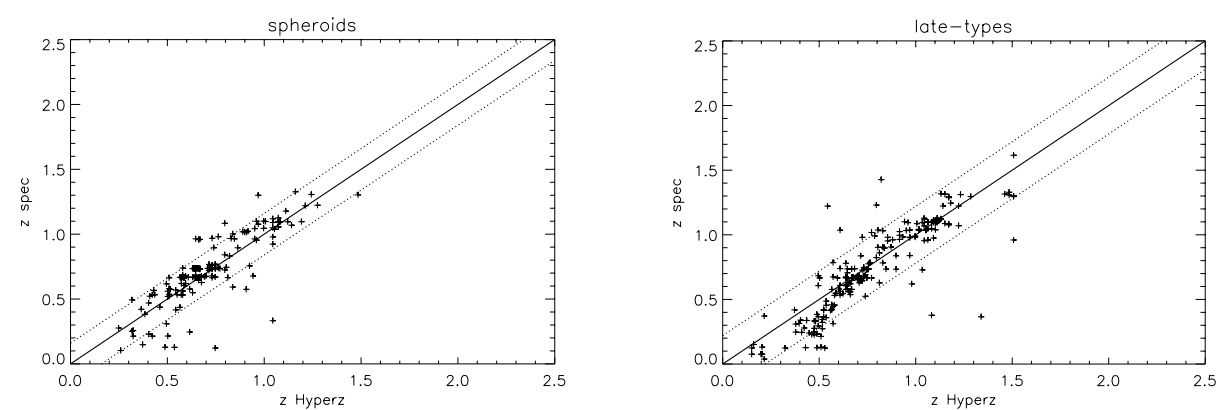

Fig. 6. Comparison of photometric versus spectroscopic redshifts for early-type (left panel) and late-type galaxies (right panel). We report only the sources with $S_{3.6}>10 \mu \mathrm{Jy}$ and those for which Hyperz found highly reliable fit (corresponding $\chi^{2}$ probability above 99\%). The dotted lines delimit the $2 \sigma$ uncertainty ranges.

Sb spirals (S b.ised). We assumed the reddening law by Calzetti (2000).

We used the numerous spectroscopic redshifts in the field to check the reliability of our photometric estimates. For the spheroid (late-type) classes, we obtained a median offset of $\Delta(z) /\left(1+z_{\text {spec }}\right)=0.06(-0.08)$ and an rms scatter of $\sigma[\Delta(z) /(1+$ $\left.\left.z_{\text {spec }}\right)\right]=0.08(0.11)$. In Fig. 6 we report a comparison of photometric versus spectroscopic redshifts separately for early-type (left panel) and late-type galaxies (right panel). We show here sources with $S_{3.6}>10 \mu \mathrm{Jy}$, for which Hyperz found a reliable fit (corresponding to a rejection probability based on $\chi^{2}$ lower than 99\%). These plots show that our procedure implies some systematic deviation at $z<0.6$, where Hyperz tends to underpredict the redshift.

For only 60 sources in the complete $10 \mu \mathrm{Jy}$ sample, either COMBO-17 or the Hyperz fits turned out to be marginally acceptable or bad (rejection $\chi^{2}$ probability higher than $95 \%$ ). This corresponds, however, to only $\sim 4 \%$ of the Spitzer complete sample, hence does not affect on our conclusions.

\section{Statistical analyses}

\subsection{Extragalactic number counts from Spitzer IRAC data}

We first estimated the extragalactic number counts at $3.6 \mu \mathrm{m}$ in the GOODS-CDFS area by weighting each source with the reciprocal of the effective area $1 / A_{\text {eff }}\left(>S_{i}\right)$ within which they could be detected to a given flux density. The errors associated with the counts in each flux level were computed as in Gruppioni et al. (2002):

$\sigma_{N}=\sqrt{\sum_{i} 1 / A_{\mathrm{eff}}^{2}\left(>S_{i}\right)}$,

where the sum is over all the sources with flux density $S_{i}$. These errors represent the Poissonian term of the uncertainties, and have to be considered as lower limits to the total errors.

We plot in Fig. 7 a comparison of the IRAC/GOODS total differential $3.6 \mu \mathrm{m}$ counts from our analysis with those published by Fazio et al. (2004), which were computed from a much wider sky area ( $\sim 9$ square degrees). We report our counts corrected for incompleteness (filled diamonds) and those uncorrected (open diamonds), versus the corresponding corrected (dotted line) and uncorrected (solid line) counts by Fazio et al. (2004).

A general agreement is observed between the two independent samples, in particular concerning the "raw" counts. Once the data are corrected for the corresponding selection functions, we observe that the Fazio et al. distribution stays slightly higher at the fainter flux range $\left(S_{3.6}<3 \mu \mathrm{Jy}\right)$. Below this level, source

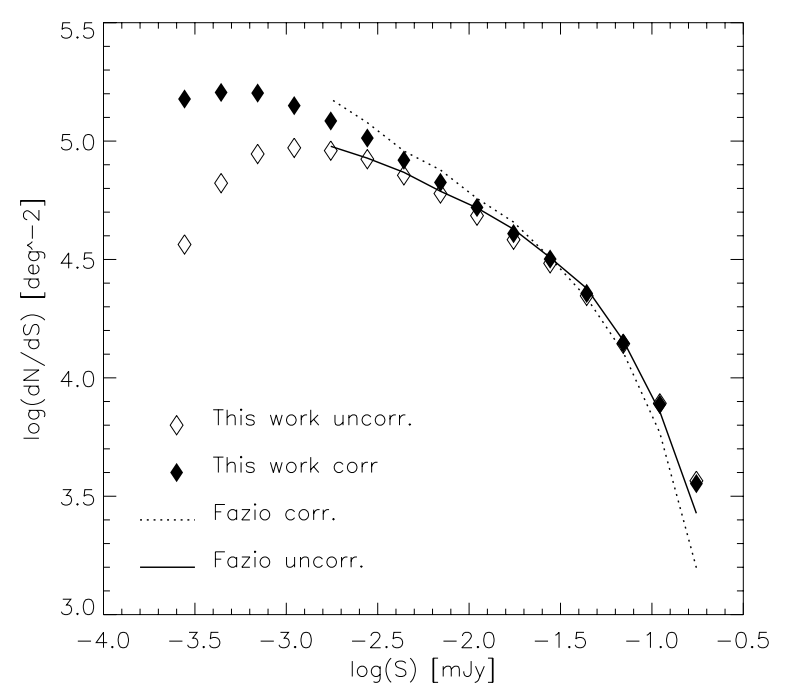

Fig. 7. The IRAC/GOODS differential $3.6 \mu \mathrm{m}$ counts from this work are compared with those published by Fazio et al. (2004). We report our incompleteness-corrected (filled diamonds) and the uncorrected (open diamonds) data with the corresponding corrected (dotted line) and uncorrected (solid line) counts by Fazio et al. (2004).

confusion starts to play some role, and it is difficult to assess if the reason for this slight discrepancy might be due to different treatment of the blended sources or differences in the determination of the completeness corrections. In any case, this does have quite a modest impact on the integral counts that are used in the following.

Figure 8 provides details about the cumulative number counts for our IRAC/GOODS sample of $3.6 \mu \mathrm{m}$ selected galaxies (empty diamonds) for different morphological classes (all stars are excluded). The contributions of each source to both the counts and the associated errors are weighted for the area within which the source is detectable.

The two upper panels in the figure show the relative contribution of the early- and late-type morphological classes, while the lower panel shows the results for the whole $3.6 \mu \mathrm{m}$ population. The error bars include both the Poisson noise and the uncertainties in the morphological classification as discussed in detail in Sect. 3. The former dominate at the brighter fluxes, while the latter uncertainty determines the error bars at the faint flux limits. All information on number counts is also reported in Table 1.

The vertical dotted lines mark the limit, $10 \mu \mathrm{Jy}$, above which the sample is $\sim 95 \%$ complete. The data and their error bars have all been corrected for incompleteness (see Sect. 2.3). The correction factor at the $1 \mu \mathrm{Jy}$ limit amounts to a factor 1.5 only (see 


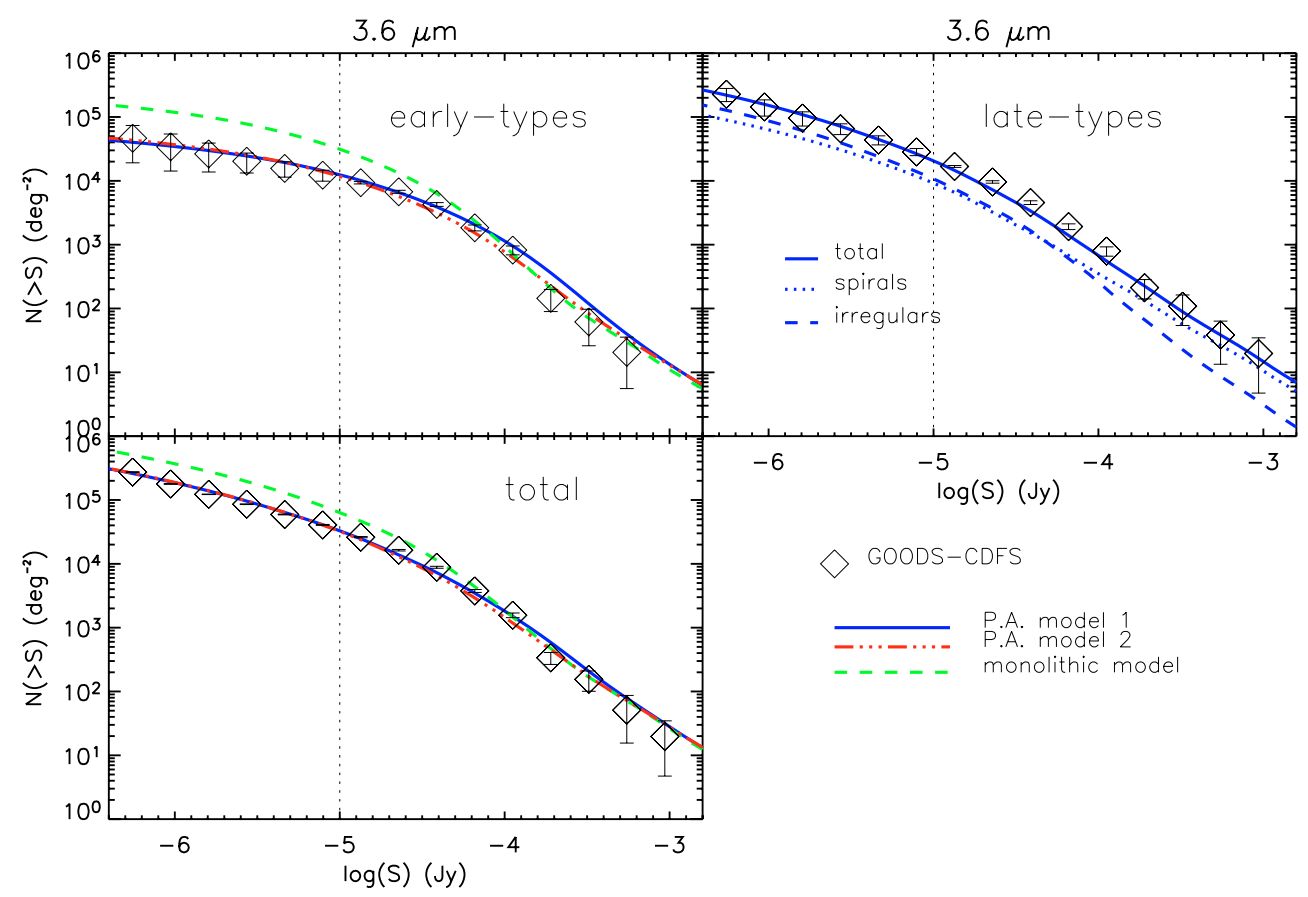

Fig. 8. The $3.6 \mu \mathrm{m}$ cumulative extragalactic number counts from the IRAC/GOODS sample, corrected for incompleteness. The two upper panels show the relative contribution of the different morphological classes (early- and late-types). The error bars include the contribution of the uncertainties in the morphological classification (see Sect. 3), which explains their increasing size at the fainter fluxes. The lower panel reports the results for the whole $3.6 \mu \mathrm{m}$ band population. The vertical dotted lines mark the $10 \mu \mathrm{Jy}$ limit, where the sample is $\sim 95 \%$ complete, but the completeness correction for the number counts is well controlled down to $\sim 1 \mu \mathrm{Jy}$ (see Fig. 1). The data are compared with the predictions of our Protracted-Assembly model 1 for spheroidal galaxies (solid blue lines), the Protracted-Assembly model 2 (three dots-dashed red line), and the monolithic model (dashed green lines).

Table 1. Galaxy number counts at $3.6 \mu \mathrm{m}$. Meaning of the columns: logarithm of the limiting flux density in Jy, number of galaxies in the survey to the flux limit, incompleteness correction factor, integral number counts in square degrees ${ }^{-1}$, number counts for spheroidal galaxies, number counts for late-type galaxies (spiral, mergers, irregulars). Uncertainties in the number counts include those from the morphological classification.

\begin{tabular}{|c|c|c|c|c|c|}
\hline $\begin{array}{c}\log S_{3.6} \\
\text { (Jy) }\end{array}$ & $n\left(>S_{3.6}\right)$ & corr. fact. & $\begin{array}{c}N_{\mathrm{TOT}}\left(>S_{3.6}\right) \\
\left(\text { sq. degree }{ }^{-1}\right)\end{array}$ & $\begin{array}{c}N_{\mathrm{E} / \mathrm{S} 0}\left(>S_{3.6}\right) \\
\left.\text { (sq. degree }{ }^{-1}\right)\end{array}$ & $\begin{array}{l}N_{\mathrm{Sp} / \mathrm{Ir}}\left(>S_{3.6}\right) \\
\left.\text { (sq. degree }{ }^{-1}\right)\end{array}$ \\
\hline-6.25 & 5955 & 2.05 & $249731 \pm 1508$ & $22608 \pm 27608$ & $227123 \pm 52786$ \\
\hline-6.02 & 5095 & 1.59 & $165716 \pm 1365$ & $21456 \pm 19930$ & $144260 \pm 40959$ \\
\hline-5.79 & 4160 & 1.36 & $115744 \pm 1206$ & $19398 \pm 12638$ & $96346 \pm 24134$ \\
\hline-5.56 & 3343 & 1.20 & $82058 \pm 1047$ & $16457 \pm 6982$ & $65601 \pm 12565$ \\
\hline-5.33 & 2431 & 1.15 & $57206 \pm 882$ & $13577 \pm 4227$ & $43629 \pm 7059$ \\
\hline-5.10 & 1757 & 1.10 & $39551 \pm 727$ & $11108 \pm 2390$ & $28443 \pm 3639$ \\
\hline-4.87 & 1195 & 1.07 & $26158 \pm 567$ & $9359 \pm 423$ & $16799 \pm 567$ \\
\hline-4.64 & 761 & 1.05 & $16351 \pm 433$ & $6739 \pm 363$ & $9612 \pm 433$ \\
\hline-4.41 & 419 & 1.03 & $8834 \pm 302$ & $4269 \pm 292$ & $4565 \pm 302$ \\
\hline-4.18 & 183 & 1.00 & $3758 \pm 199$ & $1838 \pm 195$ & $1920 \pm 199$ \\
\hline-3.95 & 78 & 1.00 & $1614 \pm 131$ & $822 \pm 130$ & $792 \pm 131$ \\
\hline-3.72 & 17 & 1.00 & $356 \pm 71$ & $144 \pm 54$ & $212 \pm 71$ \\
\hline-3.49 & 8 & 1.00 & $169 \pm 54$ & $61 \pm 35$ & $108 \pm 54$ \\
\hline-3.25 & 3 & 1.00 & $58 \pm 35$ & $20 \pm 15$ & $38 \pm 25$ \\
\hline-3.02 & 1 & 1.00 & $19 \pm 15$ & $0 \pm 0$ & $19 \pm 15$ \\
\hline
\end{tabular}

Fig. 1). The implications of the total observed counts and the separate contributions by the various morphological classes will be discussed in Sect. 7 .

\subsection{Redshift distributions}

The observed distributions of redshifts for complete galaxy samples provide a fundamental test for evolutionary models (e.g. Franceschini et al. 1998; Somerville et al. 2004; Kitzbichler \& White 2004). Figure 9 shows our estimated redshift distributions $N(z)$ for the $3.6 \mu \mathrm{m}$ sample with $S_{3.6}>10 \mu \mathrm{Jy}$ in the
CDFS area, a flux level at which the sample is $47 \%$ spectroscopically complete. The distributions here include 695 spectroscopic, 375 COMBO-17, and 408 Hyperz photometric redshifts. The upper panels show the relative contribution of spheroids and late-type galaxies, while the bottom panel reports the total distribution. The prominent peak around $z \sim 0.8$ is partly contributed by already known CDFS galaxy overdensities falling within the redshift bin (Cimatti et al. 2002; Vanzella et al. 2005; Le Fevre et al. 2004a; Adami et al. 2005). The total observed distribution shows a rapid decline above $z \sim 1.2$, and this feature is common to both morphological classes. Elliptical galaxies are 


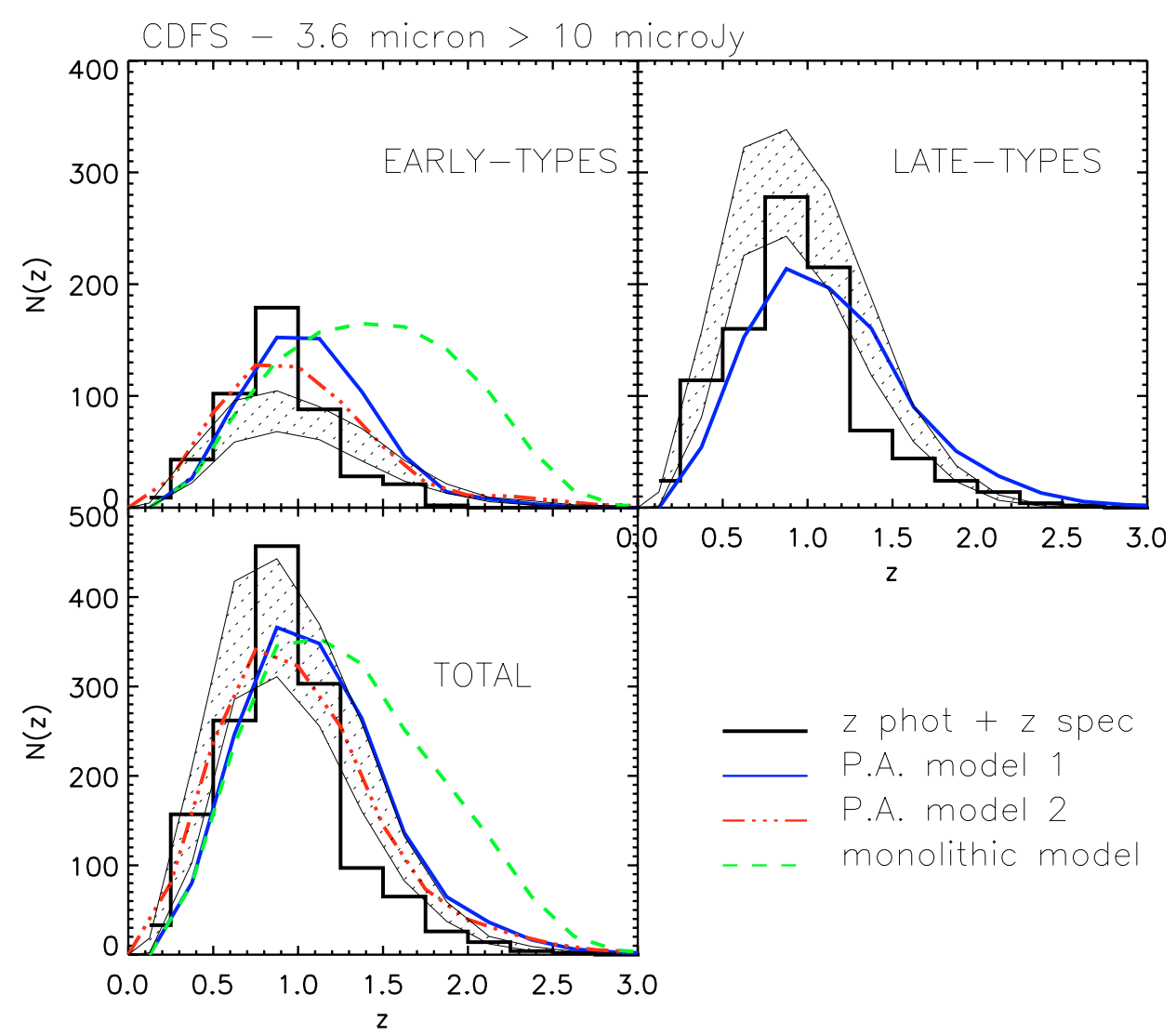

Fig. 9. Redshift distributions of the $3.6 \mu \mathrm{m}$ IRAC/GOODS sample with $S_{3.6}>10 \mu \mathrm{Jy}$ over an area of $160 \operatorname{arcmin}^{2}$, compared with model predictions. 47\% percent of the redshifts come from spectroscopic observations (Cimatti et al. 2002; Le Fevre et al. 2004a; Vanzella et al. 2005, and this paper). The upper panels show the relative contribution of spheroid and late-type galaxies, the lower panel reports the total distribution. The data are compared with the predictions of our Protracted-Assembly model 1 (solid blue lines), Protracted-Assembly model 2 (three dots-dashed red line), and our monolithic model (dashed green lines). The hatched grey regions correspond to predictions from the GALICS semi-analytical code, illustrating the effects of cosmic variance over the sampled area.

virtually absent above $z \sim 1.6$, while spiral/irregular galaxies show an apparent tail extending up to $z \sim 2.7$.

Note that the detailed behaviour of the $N(z)$, as well as of the number counts, is a combination of the intrinsic evolution of the luminosity functions, the K-corrections, and the flux limit (e.g. Ilbert et al. 2004). We will resort to detailed modellistic fits in Sect. 7 to disentangle these various effects.

Particular interest resides in the high-redshift tail of the distribution, because the statistics of the higher- $z$ galaxies provides the tightest constraints on the formation models. A question might arise here about the reliability of the morphological classification for such faint distant objects. We then spent some effort on adding visual inspection to the automatic analyses for all 183 galaxies with $z>1.3$ in the complete sample. This inspection has essentially confirmed the results of the CAS classification and has shown that $5-6 \%$ at most of the 151 late-types could be misclassified spheroids, while $\sim 30 \%$ of the 32 spheroids could instead be classified as later type galaxies. Then, on inspection, we do not expect more spheroidal galaxies at $z>1.3$ than shown in Fig. 9.

Our total observed $z$-distribution is consistent with the results of the analysis of Somerville et al. (2004) based on the photometric redshifts by Mobasher et al. (2004). The Somerville et al. analysis considered the $z$-distributions for only the total population of a $K$-band selected galaxy sample.

We also find fair consistency with a recent report by Rowan-Robinson et al. (2005) based on the analysis of SWIRE survey data (Lonsdale et al. 2004) in the Lockman verification field (VF) to $S_{3.6}>10 \mu \mathrm{Jy}$ and $r<25$, based on purely photometric redshifts and galaxy classification based on colours. Here, elliptical galaxies (red sub-population) also appear to cut off at $z \sim 1.4$, while a tail of star-forming galaxies is seen up to $z \sim 3$.

Our advantage compared to these analyses is in the substantial spectroscopic redshift coverage and the detailed structural analysis of the Spitzer galaxy sample made possible by the very deep multiband ACS data. A comparison with model predictions will be discussed in Sect. 7.

\subsection{Colour bimodality versus morphological classification}

Recent work has shown that the rest-frame colour distribution of galaxies is bimodal at all redshifts out to $z \sim 1$ (see, e.g. Hogg et al. 2002; Blanton et al. 2003; Bell et al. 2004). One peak is red and consists of mostly quiescent galaxies earlier than $\mathrm{Sa}$ in morphological type. The other peak is blue and consists primarily of SF galaxies that are later than Sa (Strateva et al. 2001; Bell et al. 2004).

With the present large dataset we are able to check if our morphological and structural classification is consistent with a colour-based selection. Figure 10 shows our observed colour distributions from the $S_{3.6}>10 \mu \mathrm{Jy}$ flux-limited sample in three redshift bins from $z=0.3$ up to $z=1.5$. Remarkably, the best colour differentiation turned out to be provided by the $V-I$ (or alternatively $V-z$ ) observed colour in all redshift bins. 

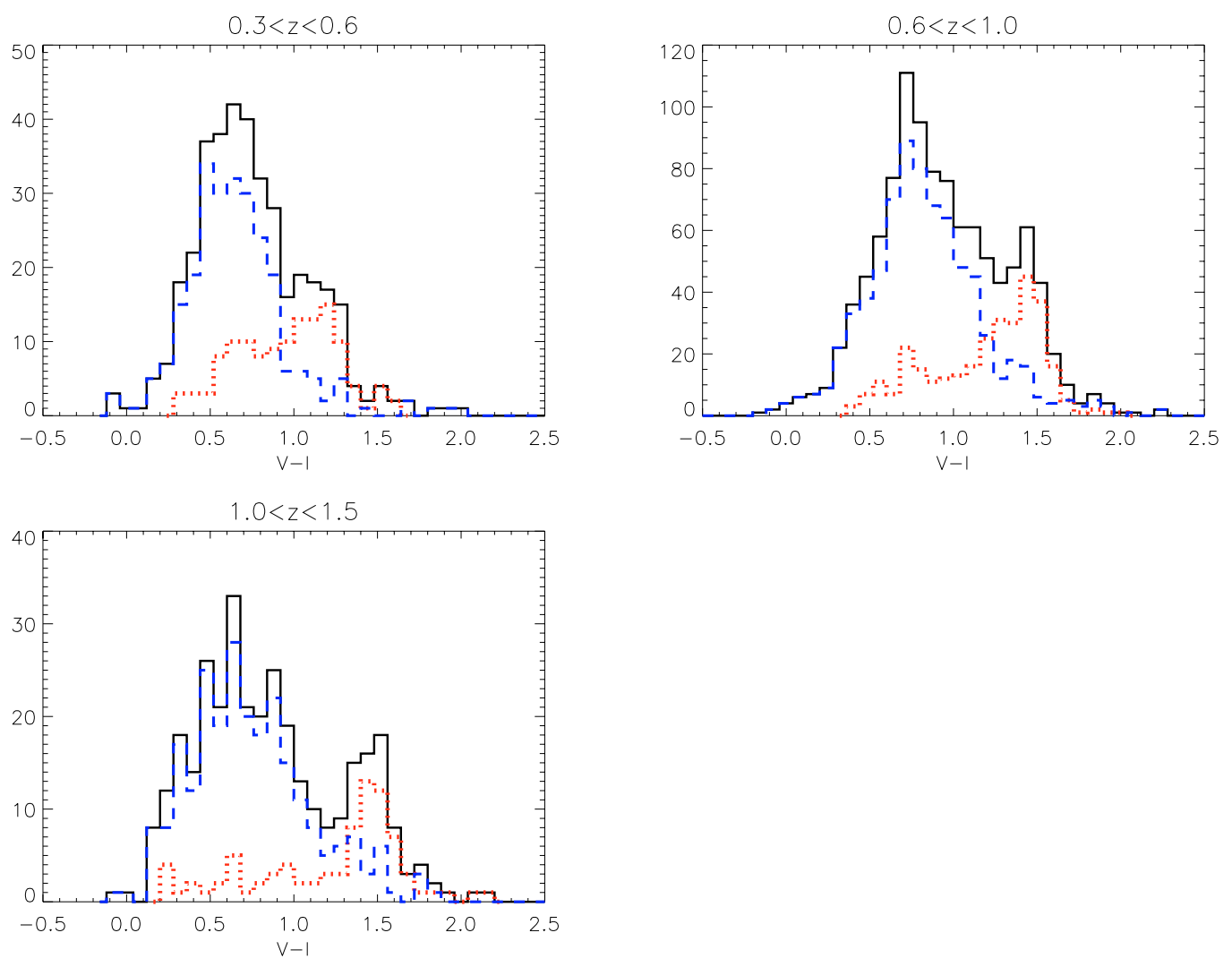

Fig. 10. Observed colour bimodality of IRAC-selected galaxies in three different redshift bins. The red dotted line and the blue dashed line correspond to our morphologically classified elliptical and late-type galaxies, respectively, showing a clear correlation between the early-type systems and the reddest ones.

These plots show not only a bimodal distribution at all redshifts, but also a clear correlation between galaxies with earlytype (late-type) morphological classification and the reddest (bluest) population. There is only a marginal trend for the earlytype population to increase the median colour with $z$, while the late-types stay remarkably unchanged. However, it is relevant to note that quite a significant fraction, on the order of $\sim 30 \%$, of the population morphologically classified as spheroidal galaxies (472 objects brighter than $10 \mu \mathrm{Jy}$ ) display blue colours typical of later morphological types. Also, this result looks largely independent of redshift.

We looked in some detail into this population of spheroidal galaxies with blue colours, in particular considering the $\sim 150$ such objects with $V-I<1$. Of these, roughly a third appear as typically bulge-dominated early-type spirals or irregulars with bright compact cores, which are misclassified as spheroidal galaxies. Roughly another third are normal ellipticals with blue colours, while the remaining third are ellipticals with signs of interactions. Less than $1 \%$ are compact objects. Our conclusion is that a structural analysis of faint galaxies provides independent information and a classification tool with respect to the most usually adopted multi-colour data.

\subsection{Further statistical constraints from deep K-band observations}

We took advantage of the deep $K$-band data in the GOODS/CDFS field to compare the statistical properties of the Spitzer long-wavelength galaxy population with those selected at $2.2 \mu \mathrm{m}$ (see Sect. 2.2). Figure 11 shows the cumulative number counts for our ISAAC/CDFS $2.2 \mu \mathrm{m}$ selected galaxy sample (thin black histogram). Stars are excluded from the analysis. The two upper panels show the relative contribution of the different morphological classes (including uncertainties in the morphological classification). The morphological analysis is the same as performed for the $3.6 \mu \mathrm{m}$ sample, and the classification criteria are consistent with those in CA05 (see Sect. 3). The bottom panel in Fig. 11 reports the results for the whole $K$ band population. The vertical dotted lines indicate the $K=21 \mathrm{mag}$ (Vega system) limit, where the sample is more than $90 \%$ complete.

We also derived redshift distributions for the $K$-band samples, taking advantage of the overlap between GOODS-CDFS, the VVDS (Le Fevre et al. 2004a), and K20 (Cimatti et al. 2002a; Mignoli et al. 2005) surveys, and of the fact that these are characterized by high spectroscopic completeness.

The results are summarised in Fig. 12, where the solid histograms in the four panels show the relative contribution of the three morphological types as classified by CA05 (including ellipticals, spirals, and irregulars/mergers, which are distinguished here from spirals). Model predictions will be compared with these data in Sect. 7.

\subsection{The space-time distribution of the sample galaxies}

We investigated the distribution in space-time of our sample galaxies further by applying the $V / V_{\max }$ test, first developed by Schmidt (1968), to study the spatial uniformity of quasars. The test compares the maximum comoving volume $V_{\max }$ within which each sample objects should be visible, with the volume $V$ occupied by the source to the observed redshift. For a uniform source space distribution, the $V / V_{\max }$ values are uniformly 


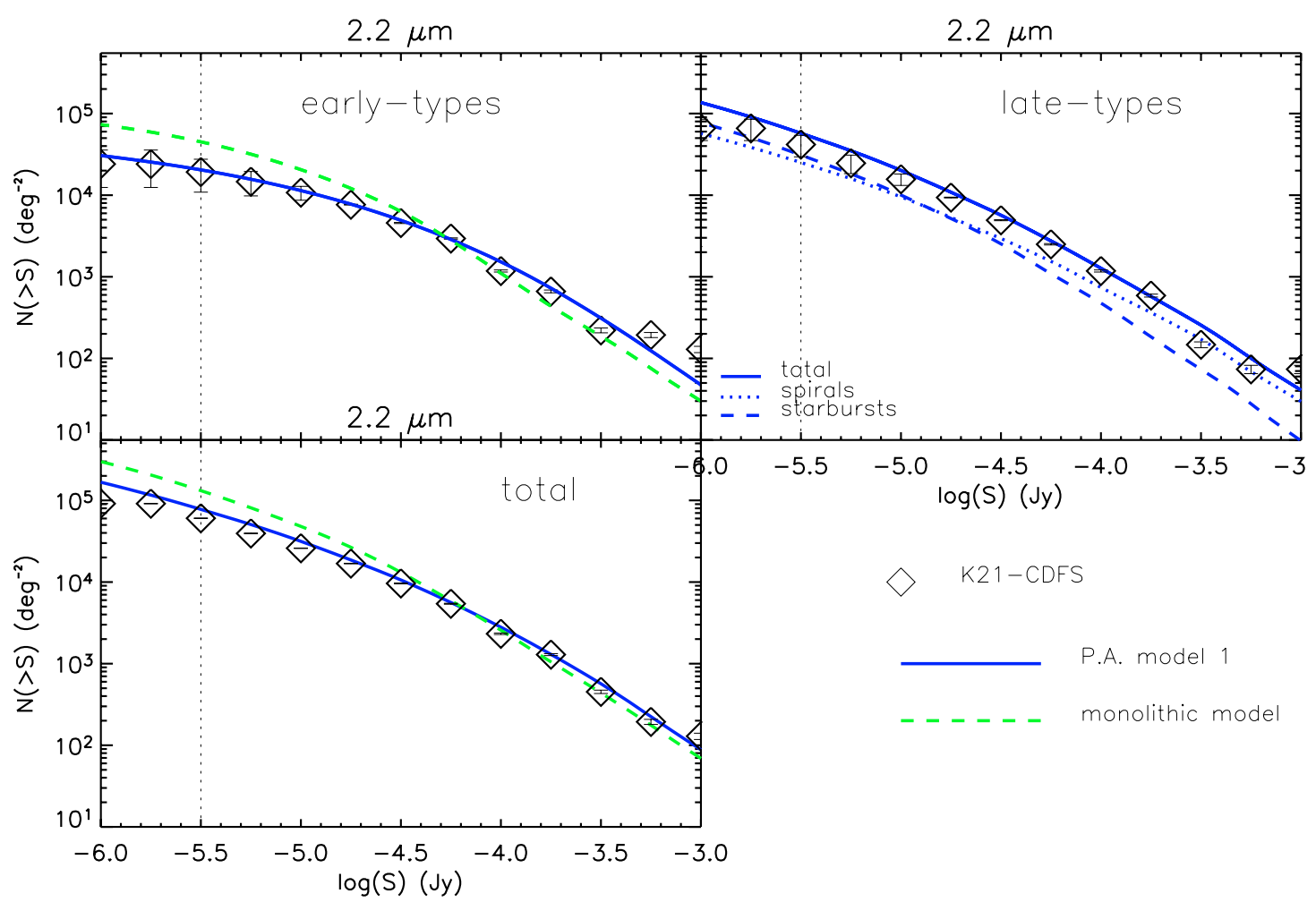

Fig. 11. $2.2 \mu \mathrm{m}$ cumulative extragalactic number counts from the IRAC/GOODS sample. The two upper panels show the relative contribution of the two different morphological classes (early- and late-types). The lower panel reports the results for the whole $K$ band population. The vertical dotted lines mark the $K=21 \mathrm{mag}$ (Vega system) limit, where the sample is more than $90 \%$ complete. The data are compared with the predictions of our PA model 1 (solid red lines).
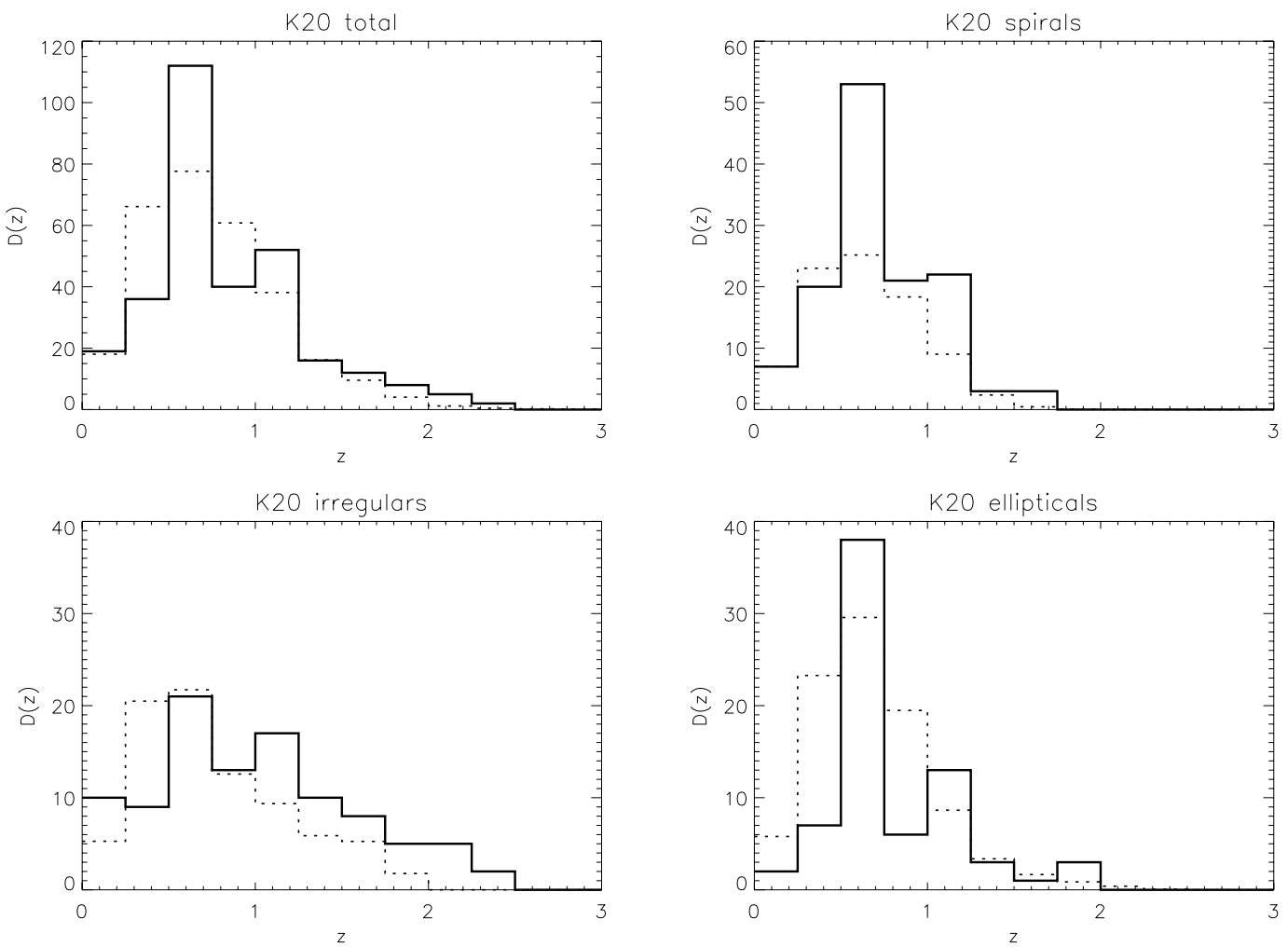

Fig. 12. Redshift distributions from the K20 catalogue, including 300 morphologically classified galaxies over an area of $32 \operatorname{arcmin}^{2}$ in CDFS (see CA05). The solid-line histograms in the four panels show the relative contributions of three morphological types as classified in CA05, the bottom left panel shows the total distribution. The dotted-line histograms mark the prediction for our best-fit evolutionary model, in particular the PA model 1 for spheroidal galaxies. 
distributed between 0 and 1 , and $\left\langle V / V_{\max }\right\rangle=0.5$ within the statistical error given by $1 /(\sqrt{12 \cdot N}), N$ being the number of objects.

The volume $V$ within the survey area $\Omega$ and the redshift $z_{1}$ is computed as

$V\left(z_{1}\right)=\Omega /(4 \pi) \int_{0}^{z_{1}} \frac{\mathrm{d} V}{\mathrm{~d} z} \mathrm{~d} z$,

where $\frac{\mathrm{d} V}{\mathrm{~d} z} \mathrm{~d} z$ is the differential comoving volume element. The volume $V_{\max } \equiv V\left(z_{\max }\right)$ refers to the maximum redshift $z_{\max }$ at which the source would still be detected with a flux density matching the survey limit. To compute it, we first obtained a best-fit spectral representation $\left(L_{v}\right)$ of the observed SED, as discussed in Sect. 6.1 below, and the best-fit rest-frame monochromatic luminosity $L_{3.6}$ (or $L_{K}$ in the case of the K20 sample discussed in Sect. 5.5.2) at the source redshift $z$. Then the redshift of the source is increased from the observed value $z$ to that at which the flux density coincides with the survey limit, using the luminosity-distance relation

$S_{v}=\frac{L_{v}}{4 \pi d_{L}^{2} K(v)}$

where $d_{L}(z)$ is the luminosity-distance at redshift $z$ and $K_{v}$ the K-correction:

$K_{v}=(1+z) \frac{\int L_{v(1+z)} T_{\nu} \mathrm{d} v}{\int L_{v} T_{\nu} \mathrm{d} v}$.

In Eq. (6) the best-fit spectral representation $L_{v}$ is kept fixed as a function of redshift, without applying any evolutionary corrections. In the case where the source luminosity $L_{v}$ would increase at higher- $z$ due to the younger ages of the stellar populations (which might be considered as typical), the volume $V_{\max }$ available to the source would be underestimated (which then provides us with an upper limit to $\left.V / V_{\max }\right)$.

\subsubsection{The $V / V_{\max }$ test for the Spitzer $3.6 \mu \mathrm{m}$ galaxy sample}

We first applied the $V / V_{\max }$ test to the objects in the flux-limited GOODS/IRAC sample with $S_{3.6} \geq 10 \mu \mathrm{Jy}$. In spite of the limited spectroscopic coverage $(47 \%)$ for the Spitzer-selected sample, we believe that we can still obtain a significant assessment of the source space distribution: the use of the mean of the $V / V_{\max }$ statistics is expected to average out the random errors (though not the systematic ones) introduced by the photometric redshift estimate.

Due to the moderate angular resolution $(\sim 3 \mathrm{arcsec})$ of the Spitzer IRAC $3.6 \mu \mathrm{m}$ images, the high-redshift detected galaxies are spatially unresolved and can be considered as behaving like point sources. For these, the effects of the cosmological angular size variations and surface brightness dimming $\left(\mu \propto[1+z]^{-4}\right)$ do not directly affect the source detectability. For this reason, we did not attempt to carry out detailed simulations of all selection effects, which is not useful in the present situation, and rather computed $z_{\max }$ by simply considering the cosmological dimming of the total flux as in Eq. (5).

A problem potentially affecting our analysis of the source space distribution are the obvious galaxy clusters/aggregations peaking at $z \sim 0.67$ and 0.73 and visible in both the VVDS (Le Fevre et al. 2004a) and GOODS/IRAC (see Fig. 16 below) catalogues, which may bias our assessment of the sample homogeneity in redshift space. For this reason and in addition to the total flux-limited sample, we considered a second one excluding sources (and the corresponding $V$ and $V_{\max }$ volumes) between $z=0.63$ and $z=0.77$. We a posteriori verified that this is a large enough redshift interval to get rid of the dispersion effect in $z$ introduced by the fraction of sources with photometric redshifts.

Figure 13 reports our results on the distribution of the $V / V_{\max }$ statistics for the spheroidal and the late-type populations as a function of the galaxy's stellar mass (see Sect. 6.1 below). The blue filled triangles correspond to sources falling in the $z=0.63$ to 0.77 redshift interval, which show a correlation between the $V / V_{\max }$ and the mass values (or with the luminosity in the subsequent Fig. 14). This correlation is due to the fact that the more massive and luminous objects at a given redshift would be visible over a wider $V_{\max }$ volume. A similar effect, but with larger scatter, is also apparent in the whole population, because of the characteristic peak in the source's redshift distribution (Fig. 9).

The mean and median values of $V / V_{\max }$ for the various galaxy subsamples are summarised in Table 2. As shown there, the $V / V_{\max }$ distributions for both the spheroidal and the SF classes show significant departures from the uniform distribution of the expectation value. The most significant effect is the apparent dearth of spheroidal galaxies at high- $z$ indicated by the very low $\left\langle V / V_{\max }\right\rangle \sim 0.32 \pm 0.03$, (median value of $\sim 0.25 \pm 0.03$ ), obtained both including and excluding the cluster volume. Although formally this departure from uniformity is very significant, we should caution however that the size of our field is relatively small and our $V / V_{\max }$ analysis subject to cosmic variance effects, particularly for the more strongly clustered spheroidal population.

Late-type galaxies show less evident departure from uniformity. Within this broad galaxy ensemble, we attempted to check if differences might be present in the $\left\langle V / V_{\max }\right\rangle$ between earlytype spirals and late-type spirals and irregulars. We used the asymmetry parameter $A$ for this and set the value of 0.4 as distinguishing between the most irregular systems from more symmetric standard spiral galaxies (e.g. CA05; Conselice 2003b). Table 2 indeed reveals that regular spirals with $A<0.4$ tend to show a somewhat similar, though less extreme, dearth of objects at the survey's upper redshift boundary, whereas merger/irregulars reveal marginal evidence of positive evolution $\left(\left\langle V / V_{\max }\right\rangle \simeq 0.513 \pm 0.005\right)$. This result is in good agreement with the value of $\left\langle V / V_{\max }\right\rangle$ in excess of 0.5 found by Daddi et al. (2004b) for star-forming (starburst and irregular) objects at $z>1.4$.

\subsection{2. $V / V_{\max }$ for galaxies in the $K$-band selected sample}

We also applied the $V / V_{\max }$ test to objects in the K20 sample $\left(K_{\mathrm{s}} \leq 20\right)$ analysed by CA05, including $74 \mathrm{E} / \mathrm{S} 0$ and 226 spiral/irregular galaxies. Though they have lower statistical significance, the advantage offered by this sample is the virtual completeness of its spectroscopic follow-up, also useful in identifying and excluding galaxies belonging to the $z=0.7$ cluster.

Even in the $K_{\mathrm{S}}$-band ISAAC images with a typical spatial resolution close to 1 arcsec, the vast majority of the sources do not appear as spatially resolved. We then calculated the $V / V_{\max }$ distribution as for the GOODS/IRAC sample, that is neglecting detailed treatment of surface brightness dimming effects. The results of the test, summarised in Fig. 14 and Table 2, appear completely consistent with those from the GOODS sample.

In conclusion, we find significant evidence in our field that galaxies belonging to the normal Hubble sequence, i.e. spheroidals and spirals with low asymmetry, tend to disappear towards the survey limit at $z \sim 1$ to 2 , while highly asymmetric objects (irregular/mergers) are more evenly distributed. Our 

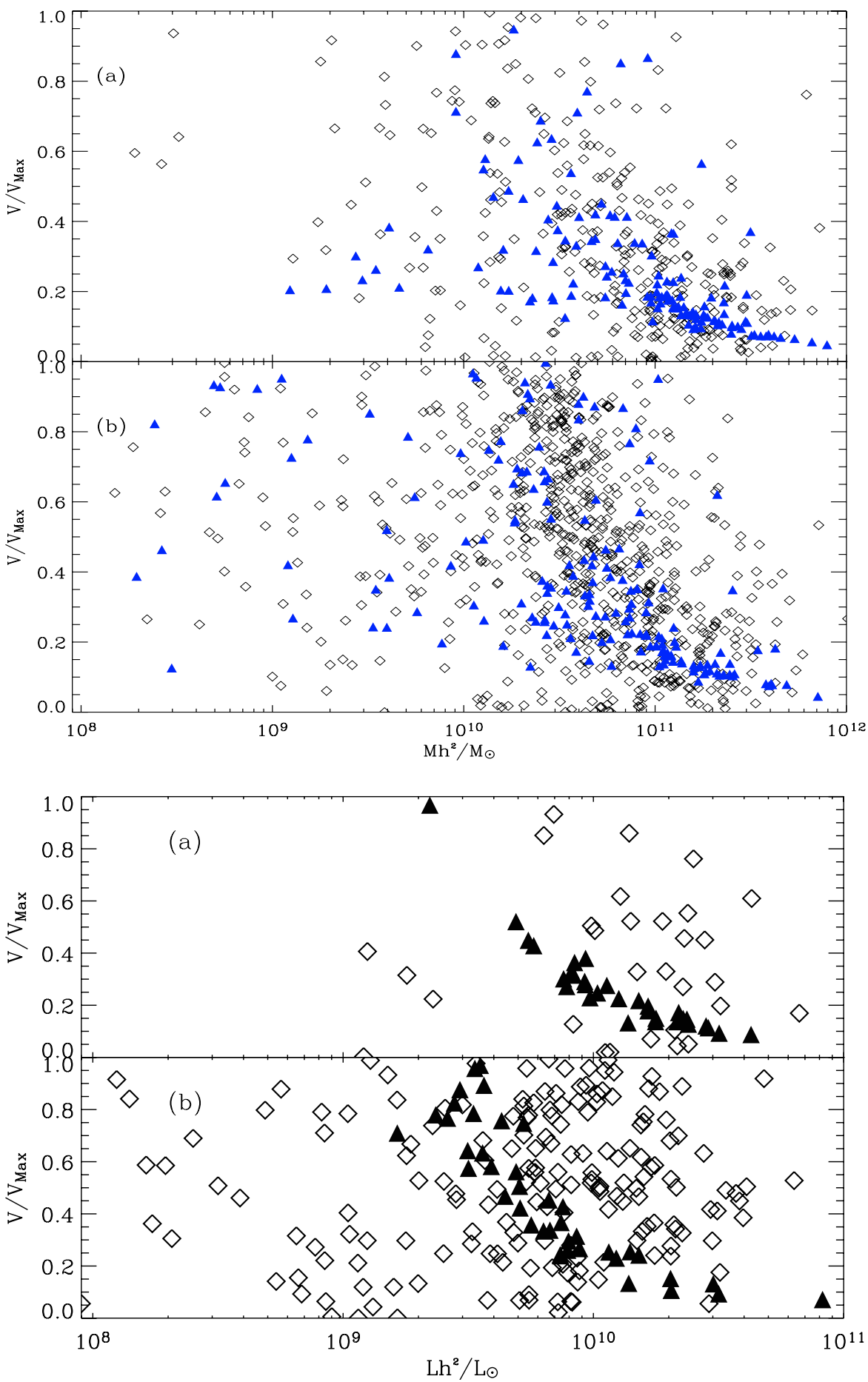

Fig. 13. $V / V_{\max }$ values for the early-type (panel a)) and late-type (panel b)) galaxies in the sample as a function of the stellar mass (estimated as discussed in Sect. 6.1) based on the flux-limited $S_{3.6}>10 \mu \mathrm{Jy}$ IRAC sample. Filled symbols indicate the objects in the vicinity of the clusters at $z=0.647$ and $z=0.735$. Mean and median values of $V / V_{\max }$ for various subsamples are reported in Table 2.
Fig. 14. $V / V_{\max }$ values for the early-type (panel a)) and late-type (panel b)) galaxies in the sample as a function of the $K$-band luminosity from the K20 sample. Filled symbols indicate the objects belonging to the cluster at $z=0.735$. Mean and median values of $V / V_{\max }$ for various subsamples are reported in Table 2. results extend to low redshifts and strengthen the conclusion of Daddi et al. (2005a) that the $V / V_{\max }$ distribution is skewed to low values for the spheroidal population, indicative of negative evolution. The characteristics of this evolution will be better specified and clarified in the following sections.

\section{The galaxy evolutionary mass and luminosity functions}

The quality of data available in this area, particularly the redshift information (either spectroscopic or photometric) and the extensive photometric coverage of the galaxy SEDs, motivated us to attempt a direct estimate of the evolutionary luminosity and mass functions for galaxies.

\subsection{The broad-band spectral fitting procedure}

Our estimate of the stellar mass follows from a detailed comparison of the observational SEDs with galaxy synthetic spectra. To generate them, we adopted the spectrophotometric synthesis code described by Berta et al. (2004), which is a development of the one described in Poggianti et al. (2001). We made use of the latter, rather than rely on the stellar mass estimated e.g. by the Hyperz code (Bolzonella et al. 2000), for various reasons. The code by Berta et al. has been specifically tailored to the stellar 
Table 2. Average values of the $V / V_{\max }$ test for various galaxy populations. Median and mean values are reported inside and outside parentheses, respectively. The three upper and three lower lines refer to the GOODS/IRAC and K20 galaxy samples, to the flux limits of $S_{3.6}=10 \mu \mathrm{Jy}$ and $K=20$, respectively. Statistics of $V / V_{\max }$ are reported here for both the total GOODS/IRAC and K20 samples (cluster incl.) and after exclusion of sources taking part in strong galaxy concentrations around $z=0.7$ cluster excluded). The latter for the GOODS/IRAC sample is achieved by excluding from the sample the cosmic volume between $z=0.63$ and $z=0.77$.

\begin{tabular}{ccccc}
\hline \hline Gal. population (GOODS) & spheroidals & late-types & late-type with $A>0.4$ & late-type with $A<0.4$ \\
\hline Number of sources & 465 & 949 & 316 & 633 \\
cluster inch. & $0.318(0.252)$ & $0.452(0.424)$ & $0.502(0.496)$ & $0.403(0.353)$ \\
cluster excl. & $0.320(0.245)$ & $0.445(0.416)$ & $0.513(0.504)$ & $0.408(0.362)$ \\
\hline Gal. population $(K 20)$ & spheroidals & late-types & late-type with $A>0.4$ & late-type with $A<0.4$ \\
\hline Number of sources & 61 & 230 & 37 & 189 \\
cluster incl. & $0.313(0.272)$ & $0.499(0.439)$ & $0.430(0.385)$ & $0.426(0.332)$ \\
cluster excl. & $0.370(0.330)$ & $0.507(0.464)$ & $0.519(0.523)$ & $0.444(0.411)$ \\
\hline
\end{tabular}
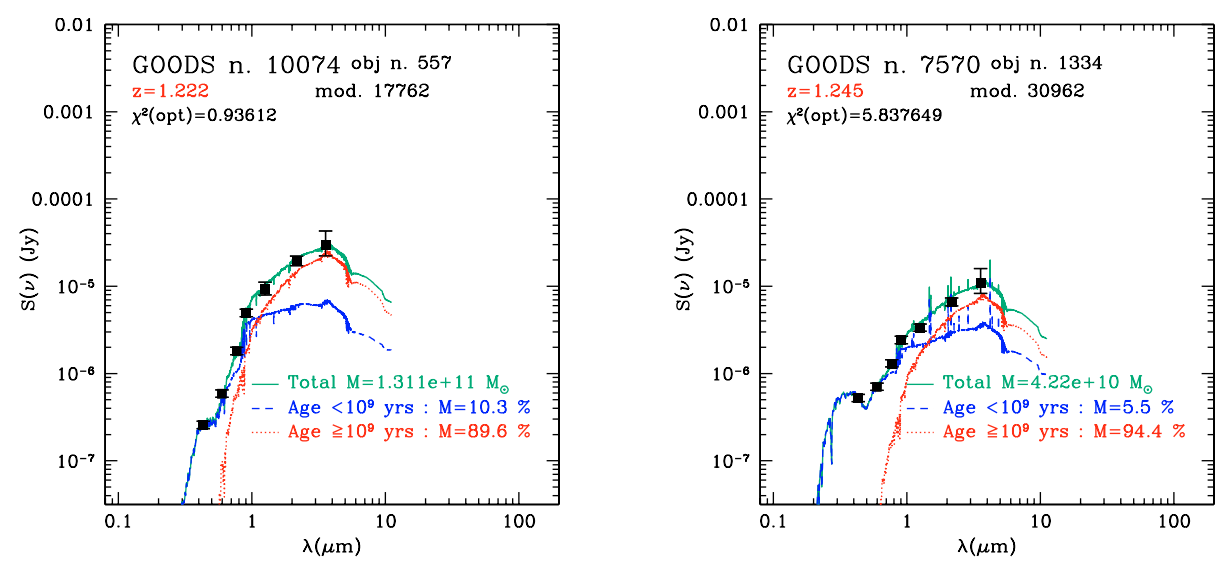

Fig. 15. Examples of spectro-photometric fits to the observed SED of a galaxy with elliptical (left-hand panel) and aone with late-type morphology. The main physical parameters are reported in the figure labels, in particular the masses of stellar populations of different ages.

mass evaluation and has been systematically tested against the outputs of other codes (e.g. Dickinson et al. 2003; Fontana et al. 2004). In addition, the code accounts in detail for the complex variety of stellar populations and population-dependent extinction typical of SF and starburst galaxies, as discussed below.

According to Berta et al., the galaxy observed SED is modelled as a combination of a set of simple stellar populations (SSP) of solar metallicity and different age. Each SSP is weighted by its total mass and extinction, and represents a temporal section in the SF history of the galaxy. So, the mass fraction contained in any SSP corresponds to a given averaged SFR during the time section covered by the SSP. Each SSP is extinguished by a different amount of dust in a uniform screen and modelled according to the standard extinction law $\left(R_{V}=\right.$ $A_{V} / E[B-V]=3.1$, Cardelli et al. 1989). The total spectrum is built up by summing the extinguished spectral energy distributions of all contributing stellar generations.

Considering that high extinction values characterize only stellar populations embedded in thick molecular clouds and that disk populations are moderately absorbed $\left(A_{\mathrm{V}} \leq 0.5\right.$, e.g. Kennicutt 1992; Kauffmann et al. 2003), we have limited the $E(B-V)$ values for the populations with ages $\geq 10^{9} \mathrm{yr}$ to be less than 0.1 .

All the SSP spectra have been computed with a Salpeter initial mass function (IMF) between 0.15 and $120 M_{\odot}$, adopting the Pickles (1998) spectral library, extended and interpolated with the Kurucz's (1993) stellar atmosphere models, following the work of Bressan et al. (1994) and Bertelli et al. (1994). Photospheric stellar and nebular (line and continuum) emission has been included through the ionization code CLOUDY
(Ferland 1996). The result is corrected for cosmological dimming and for the K-correction and compared to the observed SED by convolving with the appropriate filter transmission curves.

The code seeks the best-fit solution by exploring the parameter space through the adaptive simulated annealing algorithm (Ingber et al. 1989) and minimizing the difference between the observed data and the model measured by the $\chi^{2}$. It takes on the order of $10 \mathrm{~min}$ CPU time on a PC to explore the whole parameter space per galaxy.

The number of the SSP's involved in the fit depend on their morphological classification and redshift. Elliptical/S0 galaxies were fitted by using only SSP's older than $5 \times 10^{8}$ yr. Late-type galaxies were modelled by adopting a combination of 9 SSP's, between $1 \times 10^{7} \mathrm{yr}$ and $12 \times 10^{9} \mathrm{yr}$. Obviously, for each galaxy only the SSP younger than the age of the universe at the galaxy redshift were considered.

Our code outputs best-fit estimates for various physical parameters (e.g. rest-frame luminosity, age, SF rate, extinction, stellar mass) for each sample galaxy. Due to the wide exploration of the parameter space, we expect that not only the stellar mass value, but also the corresponding uncertainty should be fairly reliable for a given stellar IMF. This especially benefits from the Spitzer $3.6 \mu \mathrm{m}$ flux constraint on the old stellar populations dominating the stellar mass, particularly for the higher redshift $(z \geq 2)$ galaxies.

The typical $(2 \sigma)$ uncertainties in the stellar mass determination depend moderately on the source redshift (mostly thanks to the Spitzer flux constraint) and are on the order of a factor 2 (somewhat less for spheroidal galaxies in which the extinction is 


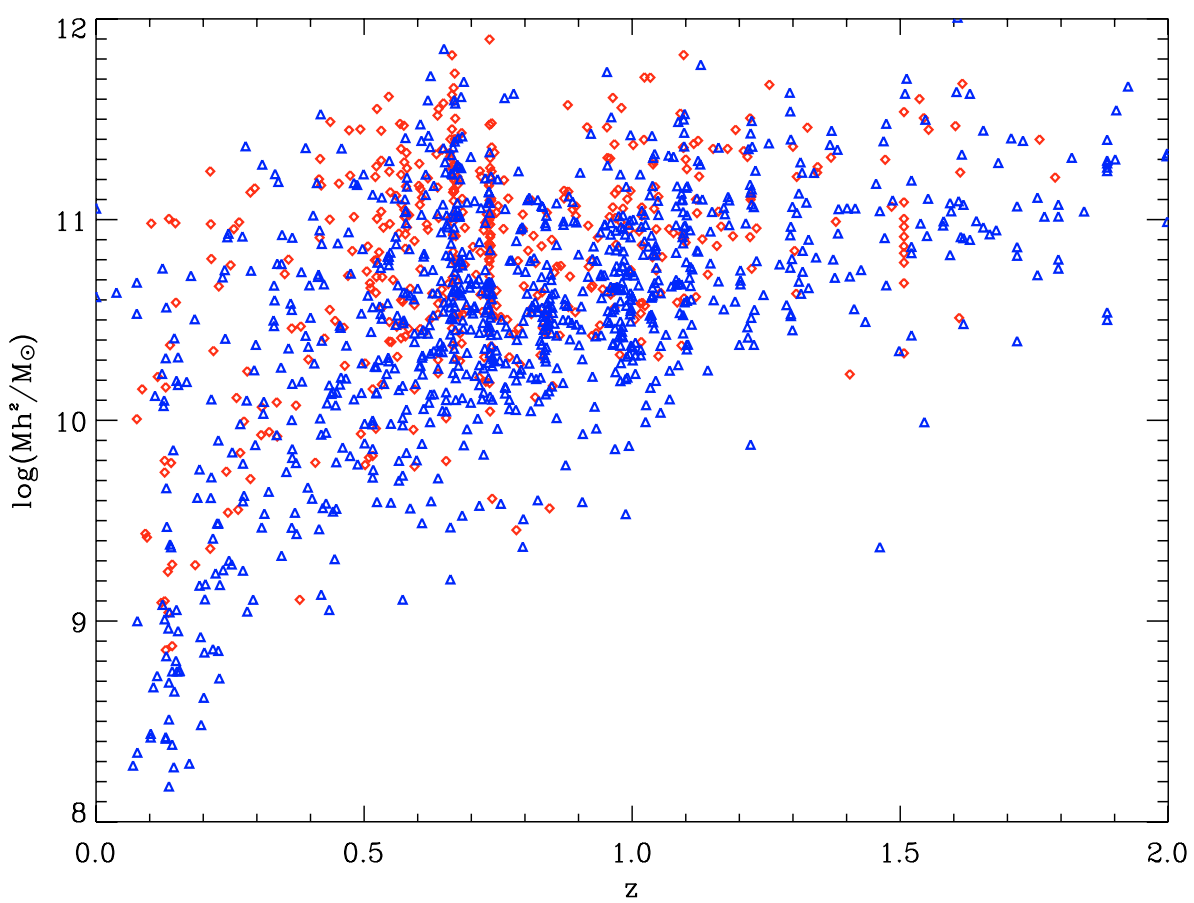

Fig. 16. Mass distribution as a function of redshift for the $3.6 \mu \mathrm{m}$ IRAC/GOODS sample with $S_{3.6}>10 \mu \mathrm{Jy}$. Morphologically classified ellipticals are marked as red diamonds, late-types as blue triangles. $47 \%$ of the redshifts have a spectroscopic confirmation. Two structures at $z \sim 0.67$ and at $z \sim 0.73$ are particularly evident.

presumed to be ineffective, and somewhat more for star-forming in which the extinction adds to the total uncertainty).

We applied our spectrophotometric fitting algorithm to the IRAC/GOODS $3.6 \mu \mathrm{m}$ sample that is brighter than $10 \mu \mathrm{Jy}$, using all the photometric bands available on the multi-wavelength catalogue (see Sect. 2.6.4), and the redshift. A couple of examples of the SED fitting quality are reported in Fig. 15, where the contributions by young and old stellar populations are explained.

In Fig. 16 we report the galactic stellar mass distribution as a function of redshift for the $3.6 \mu \mathrm{m}$ IRAC/GOODS sample with $S_{3.6}>10 \mu \mathrm{Jy}$. Morphologically classified ellipticals are marked, while the two structures already mentioned at $z \sim 0.67$ and at $z \sim 0.73$, among others, are particularly evident.

\subsection{Luminosity and mass functions}

Luminosity functions in various redshift intervals were estimated using the $1 / V_{\max }$ test, a standard method for flux limited samples (e.g. Dickinson et al. 2003; Fontana et al. 2004; Bundy et al. 2005). We followed the same procedure to estimate $V_{\max }$ as in Sect. 5.5, except for the effect of the redshift binning: when $z_{\text {low }}<z<z_{\text {high }}$, the $V_{\max }$ is calculated as:

$V_{\text {max }}=\Omega \int_{z_{\text {low }}}^{\min \left(z_{\text {high }}, z_{\max }\right)} \frac{\mathrm{d} V}{\mathrm{~d} z} \mathrm{~d} z$.

Then the comoving number of galaxies for a given redshift bin a and for a luminosity interval $\Delta L$ was estimated as:

$\Phi(L) \Delta L=\sum_{i} \frac{1}{V_{\max }^{i}} \Delta L$.

We report in Fig. 17 the luminosity functions $\left(L_{3.6}=v_{3.6} L\left(v_{3.6}\right)\right.$ calculated for three redshift bins: $0.2<z<0.55,0.55<z<0.8$ and $0.8<z<1.4$, containing 258, 471, and 503 galaxies, respectively. In each panel, the early- and late-type contributions are plotted, together with the total functions (see the figure caption for the meaning of the symbols). Error bars were estimated by Poisson statistics. We did not attempted to include in our error budget the effects of uncertainties in the photometric redshift and stellar mass estimates, which are overwhelmed by those related to the cosmic variance. We excluded redshift bins above $z=1.4$ from the analysis, since beyond this limit the spectroscopic completeness becomes low, the photometric redshifts are less reliable and the morphological classification more uncertain or even impossible. Numerical values and errors for the luminosity functions are reported in the Appendix.

To deal with the two prominent structures at $z \sim 0.67$ and $z \sim 0.73$, galaxies in the redshift interval $z=0.63$ to 0.77 have been removed, as in Sect. 5.5.

We then checked the level of agreement of our sampling compared to well-established luminosity functions. Figure $17 \mathrm{re-}$ ports in each panel the $K$-band local LF by Cole et al. (2001), transformed to $3.6 \mu \mathrm{m}$ using the rest-frame colour of a typical sample galaxy and adapted for cosmology. This transformation is rather straightforward because the spectra of galaxies are all very similar to each other in this spectral range (we find $\left\langle S_{2.2} / S_{3.6}\right\rangle \simeq 10^{0.24}$ ). We see excellent agreement between our LF in the lowest-redshift bin and the Cole et al. local LF, except for a slight excess in the highest luminosity bin, most likely due to evolution. The low- $z$ function at the higher luminosities is dominated by early-type galaxies, in agreement with Bundy et al. (2005), Kauffmann et al. (2003), and Croton et al. (2005).

The trend to increasing luminosity continues with increasing redshift and gets quite significant in the $z=1.2 \mathrm{bin}$. At this epoch our most massive galaxies are $\sim 0.7$ mag brighter on average than at $z=0$, in good agreement with the estimate by Treu et al. (2005a) based on a detailed study of the evolution of the early-type galaxy fundamental plane. A likely interpretation for this increase in luminosity may be a decrease in the $M / L$ due to the dominant stellar populations in galaxies getting younger 


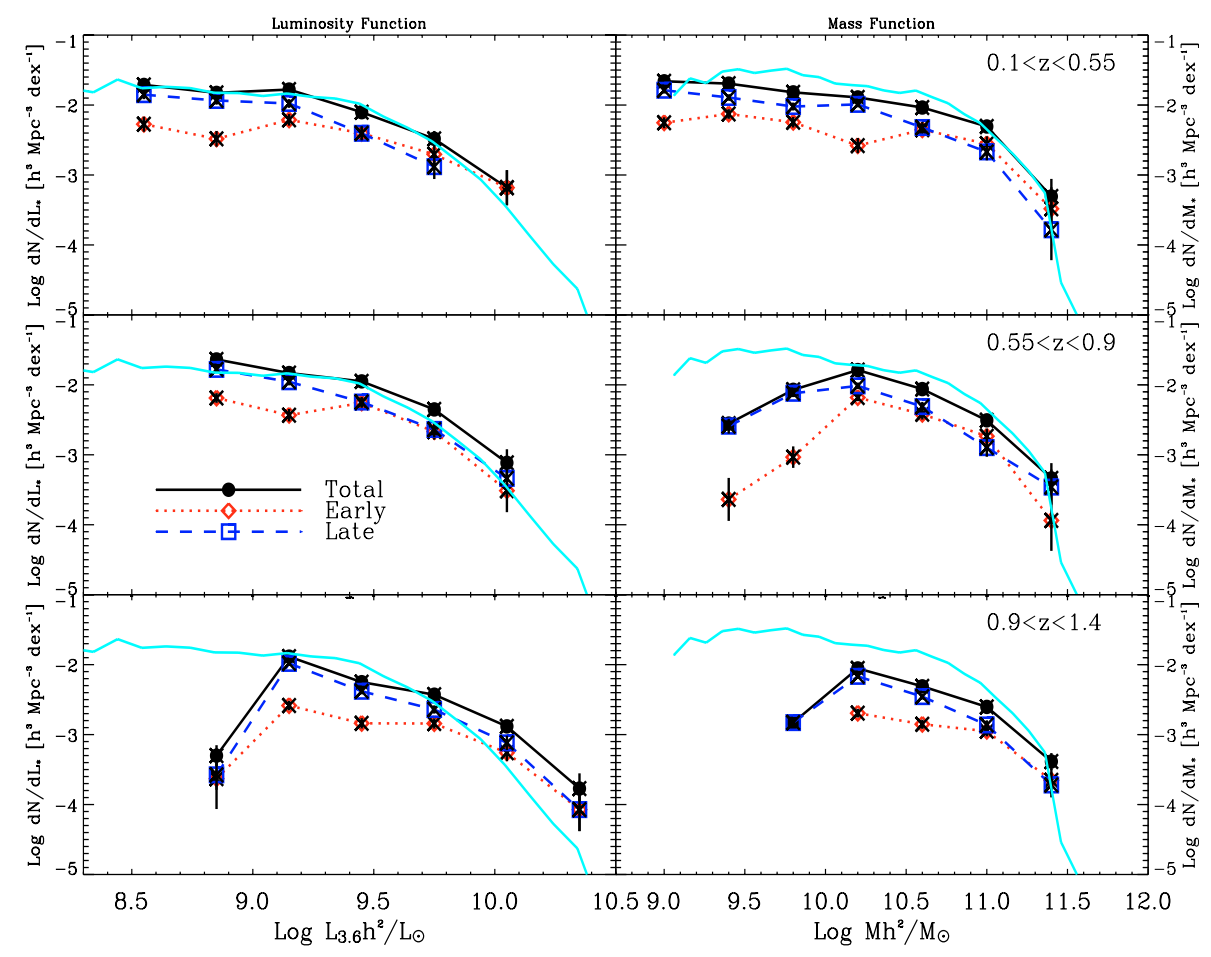

Fig. 17. Mass (right-hand panels) and luminosity (left-hand panels) function estimates derived from the $3.6 \mu$ m IRAC/GOODS sample with $S_{3.6}>10 \mu \mathrm{Jy}$, split into three redshift bins from $z=0.1$ up to $z=1.4$. The contributions of the various morphological classes are marked with different symbols: early-types (open diamonds - dotted lines), late-types (open squares - dashed lines), total (filled circles - solid lines). The thin solid line on the right marks the local mass function from Cole et al. (2001). In the intermediate redshift panels for both the mass and the luminosity functions, we excluded all sources falling in the redshift interval $0.63<z<0.77$, bracketing the cluster overdensities visible in Fig. 16 . All plotted values of the mass and luminosity functions are expressed in terms of the $h=H_{0} / 100 \mathrm{~km} \mathrm{~s}^{-1} \mathrm{Mpc}^{-1}$ parameter.

with redshift. It is hard to establish from the left panels of Fig. 17 that there might be density evolution accompanying this evolution in $L$. Note that the K-correction in the computation of the $3.6 \mu \mathrm{m}$ luminosity has an increasing effect at increasing redshift, however, we believe that the corresponding uncertainties should not appreciably affect our LF determinations, since the near-IR galaxy spectra are rather well behaved and homogeneous in the relevant wavelength interval (see Fig. 15).

The next step for obtaining the mass functions simply requires exchanging the $L$ with $M$ in Eq. (8), and binning in mass rather than in luminosity. The results of this operation are reported in the right-hand panels of the figure and in the Appendix. The local mass function by Cole et al. (2001) is also reported for reference in all panels.

In spite of the different criteria for the sample selection, our mass functions are compatible with the results by Fontana et al. (2004) and Bundy et al. (2005), who find little evolution up to $z \sim 1$. The Fontana et al. sample is $K$-band selected, has a better spectroscopic redshift coverage $(\sim 95 \%)$, but is shallower over a smaller area (only $80 \operatorname{arcmin}^{2}$ ), while the Bundy et al. sample is mostly optically selected and covers a larger area.

While the total mass function in the low-redshift bin at median $z \sim 0.3$ in Fig. 17 keeps marginally low with respect to the Cole et al. local estimate (which might indicate either a moderate evolution in comoving density already at such low redshifts or, more likely, a mismatch between Cole et al. and our stellar mass estimates), we notice an extremely good match of the low$z$ luminosity functions. We take this as supporting the reliability of our results, which is an important check when considering the uncertainties in the photometric redshift for a significant fraction of our low- $z$ sources and of the cosmic variance problem.
The evolutionary mass functions in Fig. 17 reveal some remarkable differences compared to the luminosity functions. While the latter essentially shows a migration along the $L$-axis (luminosity evolution), the mass function is more consistent with mere evolution in number density progressing from the lowest to the highest $z$-bin.

\section{Model analysis}

In this section we investigate the effectiveness of number counts and redshift distributions in further constraining the evolutionary properties of faint high-redshift galaxies.

\subsection{Simple heuristic models for galaxy counts and related statistics}

We attempt here to compare our statistical observables with simple heuristic prescriptions. In our approach, three main galaxy classes essentially are considered as dominating the near-IR selected galaxy catalogues and being characterized by potentially different evolutionary histories: spheroidal (E/S0) galaxies, quiescent spirals, and an evolving population of irregular/merger systems (hereafter the starburst population).

It is straightforward to show (e.g. based on the luminosity functions of Seyfert galaxies and quasars extrapolated from the optical) that active galactic nuclei do not significantly contribute to the extragalactic counts in the IR (Franceschini et al. 2005; Bell et al. 2005b). As a result, we did not consider the contribution of active nuclei further in our analysis.

The SED templates describing the spectral shapes at different galactic ages, which are needed for calculating the K-corrections 
and transforming the luminosity functions from one wavelength to the other, were computed using the same spectral synthesis code based on the Padova stellar isocrones as in Berta et al. (2004). Consistently with Sect. 6.1, we adopted a Salpeter IMF with a lower limit $M_{1}=0.15 M_{\odot}$ and a Schmidt-type law for the SF rate:

$\Psi(t)=v M_{\mathrm{g}}(t)$,

where $v$ is a normalization parameter (SF efficiency) and $M_{g}(t)$ the residual mass of gas at any given galactic time. During phases of active SF, stellar emission is assumed to be extinguished by dust in a uniform screen, modelled according to the standard extinction law $\left(R_{V}=A_{V} / E[B-V]=3.1\right.$, Cardelli et al. 1989). A further relevant parameter is the timescale $t_{\text {infall }}$ for the infall of primordial gas. The evolution patterns for the different galactic populations considered here are obtained with the following choices of the parameters.

For early-type galaxies, we set a quick infall timescale $t_{\text {infall }}=0.1$ Gyr and a high SF efficiency, $v=2 \mathrm{Gyr}^{-1}$. The corresponding SF law has a maximum at a galactic age of $0.3 \mathrm{Gyr}$, and is truncated at $0.8 \mathrm{Gyr}$ to mimic the onset of a galactic wind. During this quick SF phase, the galaxy emission is assumed to be extinguished by $A_{\mathrm{V}}=6$ mag.

For late-type galaxies, we adopted a longer $t_{\text {infall }}=4 \mathrm{Gyr}$ and a correspondingly lower efficiency $v=0.6 \mathrm{Gyr}^{-1}$. In this case, the peak of the SF occurs at $3 \mathrm{Gyr}$ and a galactic wind is never produced. The same parameters were assumed to reproduce the spectra of spirals and irregular galaxies. This may not be entirely representative of a galaxy during a starburst phase, but, given the spectral region considered in this work, our assumption is still a good approximation. We have then generated two grids of model spectra for both early- and late-types spanning a range of galactic ages from 0.1 to $15 \mathrm{Gyr}$.

Our assumed local luminosity functions (LLF) at $3.6 \mu \mathrm{m}$ and in the $K$-band were derived from those estimated by Kochanek et al. (2001) for both the early-type and late-type galaxy classes, based on a $K$-band selected sample taken from the Two Micron All Sky Survey (2MASS) and including 4192 low-redshift $(z \sim 0.02)$ galaxies. We made use of their luminosity functions differentiated by morphological type according to the de Vaucouleurs's parameter $T$ estimated by the authors, and adopting the value $T=-0.5$ as the boundary between spheroidal and late-type galaxies. [For spheroids the $K$-band Schechter best-fit parameters are $\alpha=-0.92, M_{K}^{*}=-23.53$ and normalization factor $n^{*}=0.45 \times 10^{-2} \mathrm{Mpc}^{-3}$ for $H_{0}=100 \mathrm{~km} \mathrm{~s}^{-1} \mathrm{Mpc}^{-1}$ ]. Transformation from 2.2 to $3.6 \mu \mathrm{m}$ was performed with the SED templates for the two classes at the present cosmic time.

In our schematic evolutionary model, we have assumed that, once formed at a given redshift, the comoving number densities of the spiral population stays constant, while the galaxy luminosities evolve according to their evolutionary stellar content. This choice reflects our assumption that, once having acquired its final morphological structure within the Hubble sequence, a normal galaxy evolves only due to the secular change of the integrated stellar spectrum.

For the spiral galaxy class, we assumed a high redshift of formation $\left(z_{\text {form }}=5\right.$, but the specific value is by no means critical, since any other choice between 2 and 5 would give essentially the same results) and constant number density henceforth. Spheroidal models are developed in Sect. 7.1.2 below.

\subsubsection{A population of fast-evolving starbursts}

We have seen in Sect. 5.5 that the $\mathrm{S}$ galaxies (the highly asymmetric, $A>0.4$, mergers/irregulars) display a $V / V_{\max }$ distribution indicative of an excess of sources at high-redshifts. This, as well as number counts and $z$-distributions, are not consistent with the assumption that the galaxy luminosity function evolves purely, following the secular evolution of the constituent stellar populations.

The inability to reproduce the faint galaxy counts in the $B$-band with no-evolution prescriptions is also a well-established result (Ellis 1997). Even more evident departures from noevolution were reported from deep observations in the mid- and far-IR (e.g. Franceschini et al. 2001; Elbaz et al. 2002; Gruppioni et al. 2002; Lagache et al. 2003). All this indicates the presence of a numerous population of irregular/merging systems at highredshifts, very likely suggesting luminosity, as well as density evolution going back in cosmic time.

We then added to our modellistic description a population of starburst galaxies whose comoving number density $\rho(z)$ evolves according to

$\rho(z) \propto \rho\left(z_{0}\right) \times(1+z)$

for $z<1$, staying constant above, and where luminosities $L(z)$ also increase as

$L(z)=L(z=0) \times \exp [k \cdot \tau(z)]$

where $\tau(z)=1-t_{\mathrm{H}}(z) / t_{\mathrm{H}}(z=0)$ is the look-back time in units of the present Hubble time $t_{\mathrm{H}}(z=0)$, and the evolution constant is $k=1.7$ for $0<z<2$, and $k=0$ at $z \geq 2$ [such that $L(z=1) \simeq$ $2.6 \cdot L(z=0)$ and $L(z=2) \simeq 4 \cdot L(z=0)]$.

For the local luminosity function of this population of irregular/merging starbursts, we have referred to the one obtained in the $B$-band by Franceschini et al. (1988), based on a local, morphologically-selected sample (from UGC), with complete spectroscopic identification. We have both appropriately transformed this LLF to the $K$-band and taken care to slightly rescale it in such a way that the sum of this with the LLF of spiral galaxies would match the $K$-band LLF by Kochanek et al. (2001).

\subsubsection{An empirical evolutionary scheme for spheroidal galaxies}

We have considered two simplified schemes of the formation of spheroidal galaxies for immediate comparison with the data, both of them tied to fit the LLF derived from Kochanek et al. (2001). The first one is a classic prescription assuming a single impulsive episode for the formation/assembly of the field ellipticals, occurring at high redshift $\left(z_{\text {form }}>2.5\right.$, e.g. Daddi et al. 2000 and Cimatti et al. 2002b), and Pure Luminosity Evolution (PLE) thereafter. We assumed a redshift of formation $z_{\text {form }}=3.0$. In this case, the birth of stars coincides with the formation of the spheroid. In the following we refer to this as the monolithic formation model.

The second model for spheroids describes a situation in which massive ellipticals form (or at least assemble) at lower redshifts through the merging of smaller units down to recent epochs. In such a case their formation is not a single coeval process, but is spread in cosmic time. We achieved this by splitting the local spheroidal galaxies into several sub-populations, each one forming at different redshifts. For simplicity, we assumed that all sub-populations have the same mass and luminosity functions and differ only for the normalizations, whose total at $z=0$ 
Table 3. Fractional contributions to the local mass function for various spheroidal galaxy sub-classes being formed at $z_{\text {form }}$, for our best-fit PA model.

\begin{tabular}{cc}
\hline \hline$z_{\text {form }}$ & $\begin{array}{c}\text { spheroidal } \\
\text { fraction }\end{array}$ \\
\hline$z_{1}=5.10$ & $5 \%$ \\
$z_{2}=3.38$ & $5 \%$ \\
$z_{3}=1.61$ & $20 \%$ \\
$z_{4}=1.37$ & $20 \%$ \\
$z_{5}=1.13$ & $20 \%$ \\
$z_{6}=0.89$ & $20 \%$ \\
$z_{7}=0.65$ & $10 \%$ \\
\hline
\end{tabular}

has to reproduce the local observed luminosity function. We emphasize that this assumption of a luminosity function building up with time by keeping a constant shape may result as oversimplified in the light of our results in Sect. 6. However we consider it as a useful reference and defer more sophisticated treatment to future papers.

We have calibrated and tested this model against deep galaxy surveys in the $K$ band (K20: Cimatti et al. 2002a,b, HDF's: Franceschini et al. 1998 and Rodighiero et al. 2001; GDDS: Abraham et al. 2004). In our current implementation, we assume 7 spheroidal sub-populations, the bulk of which $(\sim 80 \%)$ form in the redshift interval $0.9<z<1.6$, with additional fractions being produced at higher (starting from $z \sim 5$ ) and lower $z$ (down to $z \sim 0.5$ ). The detailed fractions of field ellipticals as a function of their formation redshift in our model is summarised in Table 3.

Of this seemingly hierarchical scheme, which we name as the Protracted-Assembly (PA) model, we have considered two applications. (The name PA is intended to underline that our scheme, although generically in line with the hierarchical expectations, does not provide a physical description, and at the same time is more general). In our first considered case (hereafter PA model 1) stars are assumed to be coeval to the spheroid sub-populations forming at different redshifts $z_{\text {form }}$.

We have also considered a different situation in which the structural assembly of stars in the host galaxies happens later than their formation epoch. We have then modified our previous PA scheme by assuming two different epochs, one for the birth of stars and the other for their assembly. In this second case (hereafter PA model 2) all the stars present in today ellipticals are assumed to be born at high redshift $(z=5)$, while they are dynamically assembled in the various spheroid sub-populations at the redshifts $z_{\text {form }}$ in Table 3 .

In Fig. 8 we compare the observed IRAC/GOODS $3.6 \mu \mathrm{m}$ counts with the predictions of the three models discussed above: our PA 1 (solid blue lines), the PA 2 (three dots-dashed red line), and the monolithic model (dashed green lines). These predictions differ only for their treatment of the early-type population. We note a generally good agreement of both PAy predictions for spheroids, while the monolithic model overpredicts the elliptical number counts fainter than $S_{3.6} \sim 60 \mu \mathrm{Jy}$. This excess, by a factor $>3$, is very significant all the way down to $S_{3.6} \sim 1 \mu \mathrm{Jy}$. On the other hand, we cannot discriminate within the uncertainties between the two PA solutions. Also, the source counts for latetypes are well reproduced, with a slight tendency to underpredict them in the flux range $40 \mu \mathrm{Jy}<S_{3.6}<100 \mu \mathrm{Jy}$. In general, only the two solutions including the PA description for spheroids reproduce the total source counts well (Fig. 8, bottom panel).

Similar conclusions can be derived from the redshift distributions. In Fig. 9 the observed redshift distribution of the $3.6 \mu \mathrm{m}$
IRAC/GOODS sample with $S_{3.6}>10 \mu \mathrm{Jy}$ is compared with our models. Again, the PA predictions fit the observed distribution of spheroids much better. The PA model 1 tends to slightly overpredict the number of sources above $z \sim 1.2$ (both for early- and late-type galaxies), while this excess is not present in model 2, because in this case all stellar populations are somewhat older and dimmer at the epoch of their assembly into the galaxy.

The predictions of PA model 1 for the $K$-band statistics are presented in Figs. 11 and 12 for source counts and redshift distributions, showing generally good agreement with the observations.

\subsection{Comparison with other models}

We have compared our results with an improved version of the phenomenological evolution model by Xu et al. (2003). The model reproduces the bright end of the number counts, but starts to exceed the observations below $S_{3.6}<25 \mu \mathrm{Jy}$, for both spheroidal and late-type galaxies.

Silva et al. (2005) elaborate a more physical model considering the mutual feedback between SF regions in galaxy spheroids and the active nuclei growing in their centers. This model's predictions for passive spheroids agrees well with the $3.6 \mu \mathrm{m}$ IRAC/GOODS number counts at faint fluxes, but the model foresees too many spheroidal galaxies at $S_{3.6}>100 \mu \mathrm{Jy}$ (by factors $\sim 3-5$ ) and too few spirals/irregulars (again by factors $\sim 3$ ). The model could perhaps be made more consistent with our data in case the bulk of the high- $z$ formed spheroids could be incorporated into massive spiral galaxies as their bulge components (hence escaping classification as E/S0's at low-z).

\subsubsection{Predictions of the GALICS semi-analytic code}

GALICS is a hybrid model of hierarchical galaxy formation combining large cosmological $N$-body simulations with simple semi-analytic recipes to describe the fate of the baryons within dark matter haloes (Hatton et al. 2003).

The available set of different simulated cones of universe allows one to check the effects of clustering and cosmic variance when dealing with observations covering limited sky areas. We used the GALICS database ${ }^{7}$ to simulate 10 cones of the universe, each covering an area comparable to the IRAC/GOODS survey. The comparison of GALICS predictions with the $3.6 \mu \mathrm{m}$ observed redshift distribution is reported in Fig. 9. A general agreement is observed for the total and also for the separate early- and late-type populations. As in the case of our Protracted - Assembly model 1 (see Sect. 7.1), the number of sources at $z>1.3$ is just slightly overpredicted.

The observed number counts are compared in Fig. 18 with the predicted effect of the cosmic variance shown as the hatched regions. GALICS predictions look consistent with the total number counts, with a slight excess at $S_{3.6}<10 \mu \mathrm{Jy}$ and a dearth of spheroidal galaxies at $S_{3.6}<40 \mu \mathrm{Jy}$. Within the uncertainties in the identification of the various morphological categories in the semi-analytic mock catalogues, this hierarchical code performs well for explaining the observational data.

\footnotetext{
${ }^{7}$ http://galics. cosmologie.fr/.
} 


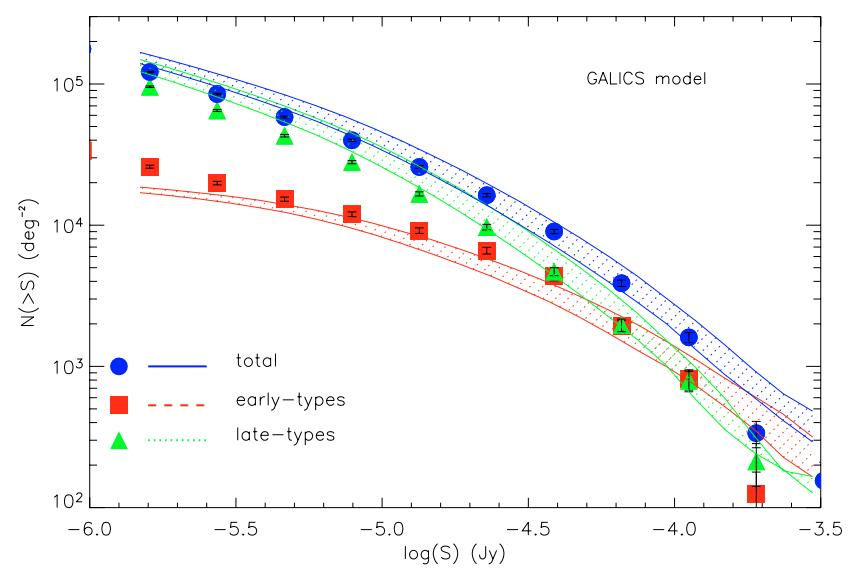

Fig. 18. The $3.6 \mu \mathrm{m}$ cumulative number counts from the IRAC/GOODS sample corrected for incompleteness compared with the predictions of GALICS, a semi-analytical code (Hatton et al. 2003). Different symbols correspond to different morphological classes (green triangles for latetypes, red squares for ellipticals, blue circles for the total). The model reports the contribution of different populations contributing to the nearIR emission (green-hatched region for late-types, red-hatched region for spheroids and a blue-hatched region for the total). The width of the hatched regions shows the effects of cosmic variance over an area of 1 square degree.

\section{Discussion}

\subsection{Phenomenological models vs. number counts and z-distributions data}

We have tried simple modellistic representations of the data on number counts and redshift distributions, to assist our interpretation. The most challenging constraints concern the spheroidal galaxy population and the modelling of their expected very luminous early phases. Results of the match between modellistic expectations and observational data are briefly discussed in the following.

\subsubsection{A Zeroth-order solution: the monolithic PLE model}

The simplest evolutionary scheme used for comparison with the data is a monolithic PLE model. Once the local LF is established by low- $z$ near-IR surveys (Sect. 7.1), this crude representation has quite a few parameters to play with and to use to correct for the inconsistencies that it revealed in the previous section. Apart from the formation epoch ( $z_{\text {form }}$ should in any case be $\sim 3$ or larger), the most important model parameters are those ruling the stellar IMF. In our application we considered the standard Salpeter form with differential spectral index $x=1.35$, $\left(N[M] \propto M^{-x}\right)$ : with this ingredient, the expected number of young spheroidal galaxies between $z \sim 1$ and 2.5 exceed the observations (see top left panel of Fig. 9).

The effects of a different choice for the stellar IMF and of variations in $z_{\text {form }}$ has been discussed in some detail by Kitzbichler \& White (2004), who compared (monolithic) PLE models with number count, $z$-distributions, and the galaxy $\mathrm{M} / \mathrm{L}$ ratios from $K$-band surveys. They find similar inconsistencies to ours above and find that, to cure them, one would need to adopt an IMF that is almost completely deprived of massive stars (with an IMF differential spectral index $x>2$ ).

We tested the effect of a slighter modification of the IMF in our monolithic model by changing from the Salpeter to the Scalo one (Scalo 1986), but the effect turned out to be a marginal reduction (by few tens percent) of the excess number counts and $z$-distributions. On the other hand, a more radical change, such as bringing the IMF spectral index $x$ to values in excess of 2, would entail problematic side effects. In particular, if we keep normalization to the local census of stellar populations in normal galaxies, a very steep IMF would prevent young galaxies from producing the metals observed in the galaxy intra-cluster plasmas (Mushotzky \& Loewenstein 1997; Baumgartner et al. 2005) and would make it impossible to explain the observed IR and optical background light (which would perhaps instead require a shallower IMF, $x<1.35$, richer in massive stars, see Madau \& Pozzetti 2000; Franceschini et al. 2001).

An alternative might perhaps be to assume that the whole early phases of the spheroidal galaxy evolution happens inside a heavily dust-extinguished medium (e.g. Franceschini et al. 1994; Kitzbichler \& White 2004; Silva et al. 2005). Such extinction should be very high (several optical magnitudes) to prevent detection by the IRAC $3.6 \mu \mathrm{m}$ band. The main difficulty with this solution, however, stems from the request by the observational constraints that such luminous galaxies should be kept enshrouded in a thick dust envelope during their whole early life, i.e. several Gyr - corresponding to the $z$ interval from $z \geq 3$ to $z \sim 1$. Considering the modest average dust attenuation in low$z$ galaxies $\left(A_{\mathrm{V}} \simeq 0.2-0.3\right.$ magnitudes, Kauffmann et al. 2003), and the results of hydrodynamical simulations of galaxy mergers (e.g. Mihos \& Hernquist 1994), it is clear that such high obscuration only characterizes transient short-lived evolutionary phases with violent redistribution of the dusty ISM on timescales of $\sim 0.1$ Gyr, like those inferred for the ultra-luminous IR galaxies (Genzel et al. 1998; Rigopoulou et al. 1999).

\subsubsection{An improved scheme: Protracted-Assembly for spheroidal galaxies}

We obtained much easier fits to the data with the alternative approach of assuming a progressive build up of the spheroidal galaxies taking place over a significant fraction of the Hubble time. We represented empirically this by splitting the luminosity function of spheroids into various sub-components and by attributing a formation redshift $z_{\text {form }}$ and a weight to each (see Table 3). For simplicity, the luminosity functions of the various sub-components were assumed to keep the same shape. Then the observational constraints are matched by a solution where $\sim 80 \%$ of the final spheroidal mass function is assembled between $z \sim 1.6$ and $z \sim 0.9$ for a standard cosmology.

We considered two specific implementations of this PA scheme: one based on the assumption that stellar populations are formed at the same time of the whole galaxy formation, $z_{\text {form }}$. The alternative was that stars existed before the galaxy was assembled, e.g. being produced at $z \sim 5$ and assembled into galaxies only later at $z_{\text {form }}$. This assumption of a progressive build-up of already aged stellar populations seems to be slightly favoured by the observations (Figs. 8 and 9).

In either case, adoption of a PA in cosmic time for spheroidal galaxies (i.e. the essential postulate of hierarchical models) appeared to overcome most of the apparent inconsistencies of the monolithic model.

The PA model is also reasonably consistent with the scanty, existing data on high- $z(z>1.4)$ spheroidal galaxies. Daddi et al. (2005a) recently colour-selected a sample in the Hubble UDF to $K \simeq 21$ and used the ACS grisms for spectroscopic identification. The number of spheroidal galaxies with $1.4<z<2.5$ over a $12 \operatorname{arcmin}^{2}$ area predicted by the PA model is perfectly consistent with the 7 objects found by Daddi et al. 
Labbé et al. (2005) combined ultra-deep $K$-band and ISAAC imaging of 5 arcmin $^{2}$ in HDF-South to identify 3 passive spheroidal galaxies with $1.9<z<3.8$ and $K<22.5$ (though one of the three could host an AGN). The prediction of the PA model at such high- $z$ depends on the detailed parameters describing the small fraction of objects forming at $z>2$ in Table 3: our best-fit predicts 1.5 galaxies for the Labbé et al. selection function.

\subsection{Evolutionary trends for luminosity and mass functions}

\subsubsection{Evolution of the global mass function}

Our investigation of the evolution of high-redshift galaxies followed two main routes. The first one was to infer general constraints from statistical observables like the number counts, $z$-distributions, and $V / V_{\max }$ analyses, as discussed in the previous section. The second approach was to derive direct information about the evolution of the main galaxy distribution functions from our reference IRAC/GOODS flux-limited sample, whose results are summarised in Fig. 17.

Do these independent lines of investigation lead to consistent solutions? We attempt to answer this question in Fig. 19, where we report the integrated comoving stellar-mass density as a function of the redshift for the two morphological classes and for the total population. The integral has been computed using galaxies with masses larger than $10^{10} h^{-2} M_{\odot}$, to ensure completeness within all three redshift bins. We find that the mass density increases by $\sim 50 \%$ from $z \sim 1.2$ to $z=0.3$ for the total sample.

Figure 19 shows the evolution of the integrated comoving mass density in spheroidal galaxies with $M h^{2}>10^{10} M_{\odot}$, as predicted by our Protracted-Assembly model (and derived from the distribution of formation epochs of the sub-populations in Table 3). Indeed, this predicted redshift dependence matches our direct determination of the evolutionary comoving density reasonably well, based on the integral of the observational mass function.

In essence, this good match provides consistent evidence that significant evolution of the global mass function has typically occurred at $z \simeq 0.7$ to 2 , also clearly indicated by the $V / V_{\max }$ analyses in Sect. 5.5. This is the most robust of our conclusions, and it agrees with the results of various other published analyses (Dickinson et al. 2003; Glazebrook et al. 2004; Fontana et al. 2004). Our average rate of evolution for the total stellar mass density $\rho_{*}$ can be approximated as an exponential fall-off with redshift

$\rho_{*}(z) \sim 6.02 \times 10^{8} h^{3} \exp \left[-\frac{(2+z)^{4}}{141.6}\right] \quad M_{\odot} / \mathrm{Mpc}^{3}$,

while for the spheroidal population a good fit to data in Fig. 19 is given by

$\rho_{*}(z) \sim 2.79 \times 10^{8} h^{3} \exp \left[-\frac{(2+z)^{5}}{342.2}\right] \quad M_{\odot} / \mathrm{Mpc}^{3}$.

Our estimated decrease by an average factor $\sim 2.5$ from $z=0$ to 1.2 of the stellar mass density of E/S0 galaxies is then somewhat steeper than the $40 \%$ decrease estimated by Treu et al. (2005a), based on previous work by Im et al. (2002). Also our observed functional-dependence on redshift, an exponential fall off, is different from their assumed power-law proportionality $\rho_{*} \propto(1+z)^{-0.6}$.

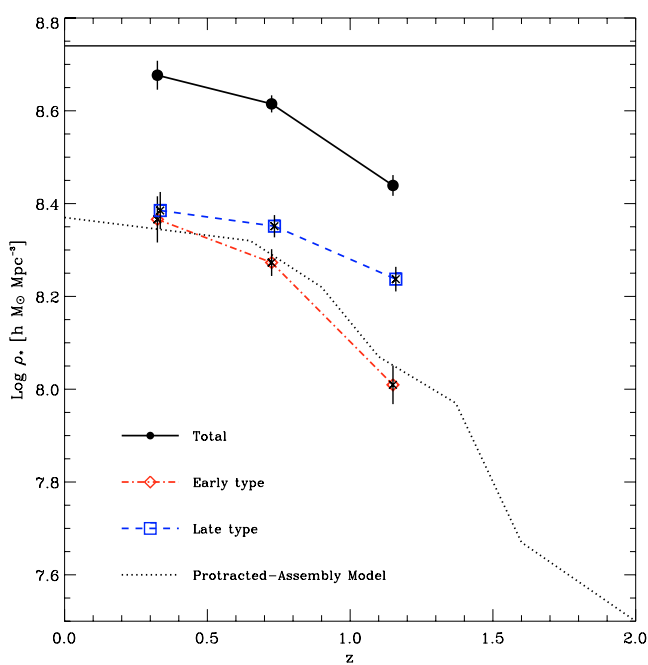

Fig. 19. Comoving integrated stellar mass density as a function of redshift, split by morphology and integrated for $M_{*} h^{2}>10^{10} M_{\odot}$. The solid horizontal line marks the local stellar mass density as measured by Cole et al. (2001) over the same mass range. This figure corresponds to a simple modification of Fig. 8 in Bundy et al. (2005), which was calculated with a higher mass cutoff $\left(M h^{2}>10^{11} M_{\odot}\right)$ : in their case, the data indicated essentially no evolution of the comoving mass density from $z \sim 0$ to $z=1$, while in our case the evolution is appreciable ( $\sim 30-40 \%$ of mass decrease over the same $z$-interval). The dotted line is the prediction of our PA model.

\subsubsection{Mass-dependent and morphology-dependent effects}

Closer inspection of our direct mass and luminosity function determinations reveals, however, quite more complexity in the evolution pattern than the simple overall decline with redshift in Fig. 19.

We report in Fig. 20 a comparison of the evolutionary mass functions at different redshifts for the two morphological categories. First of all, the evolution of the mass functions in Figs. 19 and 20 shows a differential effect with morphological type. For the late morphological types, the effect of a number-density evolution seems present but not large, while it is much more significant for the spheroidal galaxy class (right panels in Fig. 20).

An important aspect also revealed by Fig. 20 is that this decline is not uniformly shared by all galactic masses, but mostly concerns objects with $M h^{2} \leq 10^{11} M_{\odot}$. The comoving number density of the most massive galaxies, instead, stays remarkably stable from local to $z=1.2$, independently, if they belong to the spheroidal or the late-type category.

The differential effect of the evolution rate with galaxy mass applies to both morphological categories in the two panels of Fig. 20: in both cases the highest-mass galaxies show the lowest rate of evolution. Again, the differential effect is stronger for the spheroidal component.

This is in line with a similar result by Bundy et al. (2005), who find little evolution up to $z=1$ for the highest galactic masses. Indeed, Fig. 19 should be red together with Fig. 8 of Bundy et al. (2005), which shows the evolution of the same integral of the mass function, above $M h^{2}=10^{11} M_{\odot}$ : in their case there is essentially no evolution of the comoving mass density from $z=1$ to 0 , while the decrease is appreciable for both galaxy classes with our lower stellar mass threshold. The rate of galaxy assembly appears to be a strong function of the mass. This is also in keeping with the low specific SFR estimated by Daddi et al. $(2005 \mathrm{~b})$ in their most massive galaxies at $z>1.4$. 


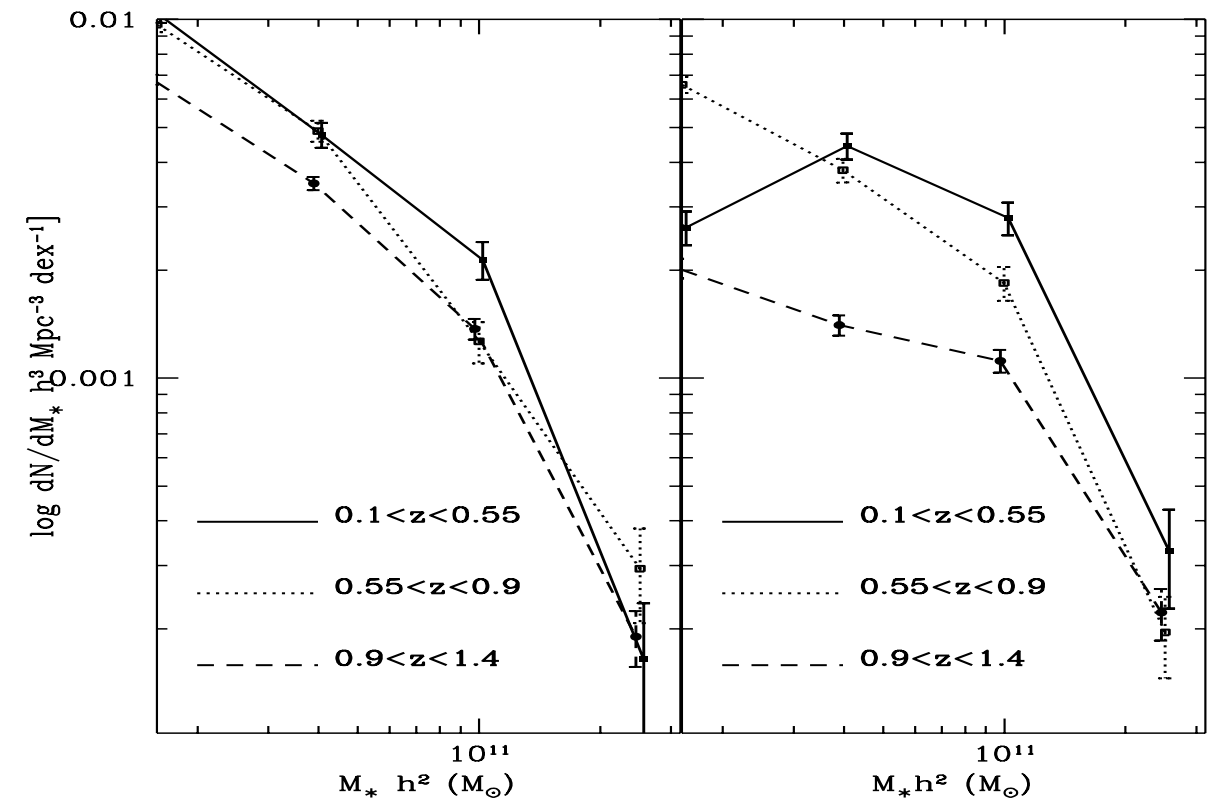

Fig. 20. The redshift-dependent mass functions for early-type (right-hand panel) and late-type (left-hand panel) galaxies in different redshift bins, as indicated in the labels. Note that the function in the $0.55<z<0.9$ interval might still suffer residual contamination by the galaxy concentrations in that redshift bin (despite galaxies from $z=0.63$ to 0.77 being excluded). (See also caption to Fig. 17.)

Treu et al. (2005a,b), Based on a high-resolution spectroscopic study of a sample of field spheroidal galaxies in the GOODS-N area, Treu et al. (2005a,b) have found significant evolution of the fundamental plane as a function of redshift and morphological properties, which can be explained as a change of the average $M / L$ ratios. They also find that this evolution depends significantly on the dynamical mass, being slower for larger masses. It is interesting to note that their analysis is based on dynamical estimates of the galactic masses. Similar conclusions are also reached by Juneau et al. (2005). As a result, this effect of differential evolution, a manifestation of the "downsizing" process originally identified and investigated by Cowie et al. (1996), appears to respond to both the mass of the galaxy's stellar content and to the total, possibly dark-matter dominated, mass. Downsizing in the formation of stars in spheroidal galaxies was also clearly indicated by Franceschini et al. (1998) from their analysis of galaxies in the HDF-North (see also hints in Gavazzi et al. 1996).

\subsubsection{Evolution patterns}

The synopsis of the evolution of the galaxy mass and luminosity functions offered by Fig. 17 may shed some light on the physical processes driving them. Let us start at low- $z$ and progress in redshift, and let us first consider the evolution of the total mass function. There is only one way to produce the number density evolution, which is observed to become effective at $z>0.9$ : this is via the intermediate-mass galaxies to progressively decrease in mass, hence migrating towards the left side of the figure. This was probably achieved through both merging of lower mass preexisting objects and formation of new stars.

That new stars are likely to be formed during the merging events accompanying the evolution of the mass function is shown by the luminosity evolution that is evident in the left-hand panels of Fig. 17. In principle, (negative) number density evolution would be expected to characterize the luminosity function in a similar way to what it does for the mass function in the right-hand panels. This negative density evolution appears completely counter-balanced by an increase in luminosity, so that the net apparent effect is that of a (positive) luminosity evolution. Clearly, if the average galaxy mass decreases and the average luminosity increases with redshift, this requires a steady decrease in the $M / L$ ratio.

Also of interest might be to try to differentiate the evolutionary paths among the different morphological classes. As for the spheroidal galaxies, two ways should produce the observed strong number density evolution: one is through the intermediate-mass objects increasing in mass, and migrating to higher masses at decreasing- $z$ (increasing cosmic time), via merging acquisition and new SF. The other path is via morphological transformations of spirals/irregulars to relaxed early-type morphologies, again a consequence of either merging episodes or gas exhaustion. All these processes are likely to happen together.

For the late-type galaxy population, and in spite of the apparent slow evolution of the mass function in Fig. 20, the evolutionary pattern was probably very complex, on one side, late-types lose in favour of the spheroidal population due to morphological transformations, on the positive side they should increase by galaxy merging and SF.

In conclusion, our analysis has confirmed that the cosmic epochs close to redshifts $\sim 1$ to 2 have experienced an active phase of SF at the low-mass end, and galaxy transformations at the knee of mass function, $M h^{2} \sim 10^{11} M_{\odot}$. Perhaps only the most massive galaxies in the field have been relatively quiet actors at these times, as shown by the correspondinlyg slow evolution of their mass function.

\subsubsection{Matching independent evidence of evolution}

Although we do not have a complete understanding of the whole set of phenomena yet, it is certainly not pure chance that the redshift interval from $\sim 0.7$ to $\sim 2$ coincides with the cosmic epochs of peak activity revealed by IR searches of the dust-obscured SF (e.g. by ISO, Franceschini et al. 2001; Elbaz et al. 2002; and by Spitzer et al. 2005; Le Floc'h et al. 2005).

In this respect, it is also interesting to note a parallel between the slow evolution to $z=1.4$ of our most massive galaxies and 
the history of the SF in the most luminous IR galaxies, which are their natural progenitors. Both the ULIRG samples analysed with Spitzer by Perez-Gonzales et al. (2005) and Le Floc'h et al. (2005) and the very luminous SCUBA sources (e.g. Smail et al. 2002; Chapman et al. 2003) show a bolometric comoving emissivity peaking at high- $z(z>1.5)$, whereas lower luminosity sources have peak emission at lower $z$. From the tight relationship of the bolometric IR emission with the SFR (e.g. Kennicutt 1998; Rowan-Robinson et al. 1997), the more luminous the IR galaxy is, the more accelerated its evolution and most confined backwards in cosmic time its main phases of SF. This meets, at least qualitatively, the requirements set by our analysis of the mass and luminosity functions.

Finally, and in light of the close relationship of quasar activity and the host galaxy formation implied by the ubiquitous presence of super-massive black holes in the cores of all local massive galaxies, it is remarkable that the same dependence on source luminosity of the cosmological evolution of the X-ray emissivity in a complete unbiassed sample of X-ray AGNs recently quantified by Hasinger et al. (2005). This similarity in the cosmic evolution of galaxies and AGNs/quasars, and the luminosity-dependent effect, were already noticed by Franceschini et al. (1999).

A large amount of independent data seems to provide concordant evidence for an evolution pattern in galaxies at $z \simeq 0.7$ to $\geq 2$ as a function of the object's mass (both stellar and total mass). These data, suggestive of a global "downsizing" in galaxy formation with cosmic time, require some important correction factors (probably related to feedback by forming objects) to the hierarchical dark matter assembly, which otherwise makes an appropriate baseline scenario, as confirmed here.

\section{Conclusions}

This paper has been devoted to a systematic exploitation of public multi-wavelength data from the GOODS survey in the Chandra Deep Field South to derive observational constraints on the emergence of the Hubble galaxy morphological sequence throughout cosmic time. Critical data for this purpose are made available, in particular, by the very deep multi-colour high-resolution imaging by HST/ACS and by the Spitzer Space Telescope deep photometric infrared imaging. We also made use of extensive optical spectroscopic observations by the ESO VLT/FORS2 and VIMOS spectrographs.

Our main selection of faint high-redshift galaxies is based on deep images by IRAC on Spitzer. We selected from them a highly reliable IRAC $3.6 \mu \mathrm{m}$ sample of 1478 galaxies with $S_{3.6} \geq 10 \mu \mathrm{Jy}$ for detailed statistical analyses and for the derivation of mass and luminosity functions in bins of redshift. We also extended the morphologically-differentiated number counts down to a flux limit of $S_{3.6}=1 \mu \mathrm{Jy}$. We carefully analysed and thoroughly tested these data for completeness and reliability, based on simulations.

Forty-seven percent of the sample objects have spectroscopic redshift from the VVDS, K20, and GOODS projects. For the remaining, we used photometric redshifts from COMBO-17 for galaxies below $z \sim 1$, while, for galaxies for which the COMBO-17 guess was above 1, we re-estimated the photometric redshifts with Hyperz. Deep $K$-band VLT/ISAAC imaging in the field is also used to derive further complementary statistical constraints and to assist the source identification and SED analysis.

This very extensive dataset is then used to assess evolutionary effects in the galaxy's stellar mass and luminosity functions, while luminosity/density evolution is further constrained with the number counts and redshift distributions. The estimate of galaxy stellar masses benefits in particular from the constraint set by the IRAC $3.6 \mu \mathrm{m}$ flux on the number of low-mass stars. The deep ACS imaging has allowed us to differentiate these evolutionary paths by morphological type, which our simulations show to be reliable at least up to $z \sim 1.5$.

The main results of the paper are hereby summarised.

- We derived luminosity functions at $3.6 \mu \mathrm{m}$ for various galaxy populations as a function of redshift up to $z=1.4$. After careful calibrations of the $M / L$ ratio, based on a detailed spectral fitting analysis to the observed SED's for each sample galaxy, we also obtained estimates of the evolutionary stellar mass functions. On one side, the $3.6 \mu$ m luminosity functions that we have derived show evidence of a positive, moderate luminosity evolution as a function of redshift (by $\sim 0.7 \mathrm{mag}$ in the $L$-band from $z=0$ to 1.2 for the most massive galaxies, $M h^{2}>10^{11} M_{\odot}$, in agreement with Treu et al. 2005a), likely due to stellar ages in galaxies becoming younger at increasing $z$. On the other hand, the corresponding global mass function shows evidence of an exponential decrease in the comoving density of galaxies $\left(\rho_{*} \propto \exp \left(-[2+z]^{4} / 141.6\right)\right)$ at the corresponding redshifts.

- The galaxy number counts, z-distributions, the $\left\langle V / V_{\max }\right\rangle$ test, as well as our direct estimate of the stellar mass function above $M_{*} h^{2}=10^{10} M_{\odot}$, provide consistent evidence of a progressive dearth (by a factor $\sim 2.5$ by $z=1.2$ for the stellar mass density, see Fig. 19 and Eq. (13)) of the spheroidal galaxy population to occur starting at low- $z$ and becoming quite significant at $z \geq 0.7$, paralleled by an increase in luminosity (half a mag in $L$-band). Simple evolutionary models, fitting the fast convergence of the number counts and redshift distributions, and the evolutionary mass function, require the main episodes for spheroidal build-up (of either old or newly-formed stellar populations) to happen between $z \sim 2$ and $z \leq 1$ for such field population, on average.

- This decrease in the comoving density of galaxies with redshift shows, however, a remarkable dependence on galaxy mass, which is strong for moderate-mass, but almost absent until $z=1.4$ for high-mass galaxies, thus confirming previous evidence of a "downsizing" effect in galaxy formation (e.g. Cowie et al. 1996; Franceschini et al. 1998). By comparison with dynamical studies of the high-redshift spheroidal population (Treu et al. 2005a,b), it is concluded that both stellar mass and total "dynamical" mass are driving parameters of this differential evolution. This evolutionary pattern may also help to explain some inconsistencies in the evolution of galaxies at the high redshifts previously reported by different teams. Our results appear consistent with recent reports by independent teams and selection functions (Fontana et al. 2004; Bundy et al. 2005).

- As for the complementary class of actively SF (irregular/merger) galaxies, deep Spitzer/IRAC $3.6 \mu \mathrm{m}$ and $K$-band observations show them as evolving towards moderately higher luminosities and number densities up to $z \sim 1$ to 2 , while normal spirals (those with asymmetry indices $A<0.4$ ) show similar, though slower, convergence at $z>1$ to that of spheroids.

- Our favoured interpretation of the estimated mass functions and evolutionary trends for the two broad galaxy categories is that of a progressive morphological transformation (due to gas exhaustion and, probably, merging) from the starforming to the passively evolving phase starting at $z \geq 2$ and continuing on down to $z \sim 0.7$. The rate of this process 
Table A.1. Redshift-dependent luminosity functions for galaxies. Luminosities are $\log \left(L_{3.6} \mu \mathrm{m} * h^{2} / L_{\odot}\right)$. Luminosity functions are in units of $\log (\mathrm{d} N / \mathrm{d} L)\left[h^{3} \mathrm{Mpc}^{-3} \operatorname{dex}(L)^{-1}\right]$, where $h=H_{0} / 100 \mathrm{~km} \mathrm{~s}^{-1} \mathrm{Mpc}^{-1}$.

\begin{tabular}{cccccccc}
\hline \hline$z$-interval & $\log L$ & tot & tot_err & early & early_err & late & late_err \\
\hline $0.1<z<0.55$ & 8.5500 & -1.7126 & -2.9737 & -2.2738 & -3.2414 & -1.8521 & -3.0475 \\
& 8.8500 & -1.8263 & -3.1048 & -2.4829 & -3.4332 & -1.9344 & -3.1588 \\
& 9.1500 & -1.7782 & -3.0808 & -2.2119 & -3.2977 & -1.9778 & -3.1806 \\
& 9.4500 & -2.1027 & -3.2431 & -2.4038 & -3.3936 & -2.4038 & -3.3936 \\
& 9.7500 & -2.4829 & -3.4332 & -2.7048 & -3.5441 & -2.8809 & -3.6322 \\
$0.55<z<0.9$ & 10.0500 & -3.1819 & -3.7827 & -3.1819 & -3.7827 & -12.0000 & - \\
& 8.8500 & -1.6367 & -3.0290 & -2.1886 & -3.3444 & -1.7797 & -3.0868 \\
& 9.1500 & -1.8317 & -3.1850 & -2.4337 & -3.4860 & -1.9566 & -3.2475 \\
& 9.4500 & -1.9506 & -3.2445 & -2.2576 & -3.3980 & -2.2457 & -3.3920 \\
& 9.7500 & -2.3515 & -3.4449 & -2.6678 & -3.6031 & -2.6378 & -3.5881 \\
& 10.0500 & -3.1150 & -3.8267 & -3.5129 & -4.0256 & -3.3368 & -3.9376 \\
$0.9<z<1.4$ & 8.8500 & -3.3009 & -4.1274 & -3.6304 & -3.9926 & -3.5752 & -4.2433 \\
& 9.1500 & -1.8855 & -3.3934 & -2.5846 & -3.7524 & -1.9823 & -3.4394 \\
& 9.4500 & -2.2509 & -3.6711 & -2.8391 & -3.9674 & -2.3806 & -3.7352 \\
& 9.7500 & -2.4292 & -3.7637 & -2.8422 & -3.9702 & -2.6413 & -3.8697 \\
& 10.0500 & -2.8824 & -3.9903 & -3.2598 & -4.1790 & -3.1185 & -4.1083 \\
& 10.3500 & -3.7717 & -4.4349 & -4.0727 & -4.5854 & -4.0727 & -4.5854 \\
\hline
\end{tabular}

Table A.2. Redshift-dependent stellar mass functions for galaxies. Masses are $\log \left(M * h^{2} / M_{\odot}\right)$. The mass functions are in units of $\log (\mathrm{d} N / \mathrm{d} M)$ $\left[h^{3} \mathrm{Mpc}^{-3} \operatorname{dex}(M)^{-1}\right]$, where $h=H_{0} / 100 \mathrm{~km} \mathrm{~s}^{-1} \mathrm{Mpc}^{-1}$.

\begin{tabular}{cccccccc}
\hline \hline$z$-interval & $\log M / M_{\odot}$ & tot & tot_err & early & early_err & late & late_err \\
\hline $0.1<z<0.55$ & 9.0000 & -1.6605 & -3.0617 & -2.2536 & -3.3592 & -1.7885 & -3.1163 \\
& 9.4000 & -1.6934 & -3.1244 & -2.1280 & -3.2883 & -1.8924 & -3.2259 \\
& 9.8000 & -1.8183 & -3.1678 & -2.2461 & -3.3684 & -2.0213 & -3.2657 \\
& 10.2000 & -1.8914 & -3.2922 & -2.5798 & -3.5441 & -1.9910 & -3.3735 \\
& 10.6000 & -2.0358 & -3.2721 & -2.3526 & -3.4305 & -2.3216 & -3.4150 \\
& 11.0000 & -2.3068 & -3.4076 & -2.5535 & -3.5310 & -2.6700 & -3.5892 \\
$0.55<z<0.9$ & 11.4000 & -3.3068 & -3.9076 & -3.4829 & -3.9957 & -3.7840 & -4.1462 \\
& 9.4000 & -2.5541 & -3.5767 & -3.6378 & -4.1506 & -2.5915 & -3.5676 \\
& 9.8000 & -2.0710 & -3.3666 & -3.0336 & -3.8462 & -2.1211 & -3.3914 \\
& 10.2000 & -1.7891 & -3.2470 & -2.1817 & -3.4648 & -2.0146 & -3.3440 \\
& 10.6000 & -2.0610 & -3.3616 & -2.4203 & -3.5418 & -2.3107 & -3.4858 \\
& 11.0000 & -2.5075 & -3.5854 & -2.7347 & -3.6990 & -2.8975 & -3.7804 \\
$0.9<z<1.4$ & 11.4000 & -3.3368 & -4.0000 & -3.7089 & -4.3011 & -3.5317 & -4.0625 \\
& 9.8000 & -12.0000 & - & -12.0000 & - & -2.8285 & -3.9854 \\
& 10.2000 & -2.0545 & -3.5966 & -2.6938 & -3.8967 & -2.1677 & -3.6564 \\
& 10.6000 & -2.3103 & -3.7603 & -2.8522 & -4.0340 & -2.4572 & -3.8326 \\
& 11.0000 & -2.6040 & -3.9127 & -2.9520 & -4.0865 & -2.8627 & -4.0421 \\
& 11.4000 & -3.3847 & -4.3039 & -3.6536 & -4.4383 & -3.7205 & -4.4718 \\
\hline
\end{tabular}

appears to depend on galaxy mass, being already largely concluded by $z \sim 1.4$ for the most massive systems.

- We finally discuss how well this evidence of a differential rate of galaxy build up with galactic mass compares with estimates of the SFR history based on deep far-IR surveys (e.g. Perez-Gonzales et al. 2005). A match between the two complementary views of the history of SFR by the best SF tracer (the bolometric flux), on one hand, and the rate of stellar mass build up traced by the near-IR emission on the other, would be achieved just by assuming that the progenitors of the most massive galaxies are the most (bolometrically) luminous sources at high-z. Ample evidence is accumulating in favour of the latter.

If the evolution pattern for galaxies to $z \sim 1.4$ is now close to being understood, the knowledge of what happened exactly in the critical higher redshift era is still limited by very poor statistics in the number of detected sources and by the lack of spectroscopic follow-up. How in detail is the mass function behaving at such high redshifts? Does the "downsizing" trend continue there, as it might seem natural to expect? The sensitivity of Spitzer/IRAC should allow accurate stellar mass determinations at these high- $z$, but much further substantial effort with powerful spectrographs is needed before we get credible answers. It is encouraging that much along this line has already been undertaken (among others, by GDDS, Abraham et al. 2004; Juneau et al. 2005; FIRES, Franx et al. 2003; GMASS, Cimatti et al., in progress; COSMOS \& z-COSMOS, Scoville et al., Lilly et al., in progress).

Acknowledgements. This work is based on observations made with the Spitzer Space Telescope, which is operated by the Jet Propulsion Laboratory, California Institute of Technology under NASA contract 1407. Support for this work, part of the Spitzer Space Telescope Legacy Science Program, was provided by NASA through an award issued by the Jet Propulsion Laboratory, California Institute of Technology under NASA contract 1407. ACS was developed under NASA contract NAS 5-32865, and this research was supported by NASA grant NAG5-7697. We are grateful for an equipment grant from Sun Microsystems, Inc. The Space Telescope Science Institute is operated by AURA Inc., under NASA contract NAS5-26555. Many of the observations leading to these results were carried out using the Very Large Telescope at the ESO Paranal Observatory. This work makes use the GalICS/MoMaF Database of Galaxies (http://galics.iap.fr). We thank L. Silva for making available to us her code's results in tabular form and thank her, Alvio Renzini, and Andrea Cimatti for useful comments. We also thank C. Gruppioni for making available to us her IDL procedures for the optical identifications. We finally warmly thank Laurence Tresse, the referee, for a careful reading of the paper and numerous useful comments. 


\section{Appendix A: Numerical values for the luminosity and mas functions}

We report numerical values of our estimated luminosity and mass functions in Tables A.1 and A.2. For ease of comparison with previous work, the values of the functions, luminosities and masses are reported in terms of the parameter $h=$ $H_{0} / 100 \mathrm{~km} \mathrm{~s}^{-1} \mathrm{Mpc}^{-1}$.

\section{References}

Abraham, R. G., Tanvir, N. R., Santiago, B. X., et al. 1996, MNRAS, 279, 47 Abraham, R. G., Glazebrook, K., McCarthy, P. J., et al. 2004, AJ, 127, 2455

Adami, C., Mazure, A., Ilbert, O., et al. 2005, A\&A, 443, 805

Baugh, C. M., et al. 2005, MNRAS, 356, 1191

Baumgartner, W. H., Loewenstein, M., Horner, D. J., \& Mushotzky, R. F. 2005, ApJ, 620, 680

Bell, E. F., Wolf, C., Meisenheimer, K., et al. 2004, ApJ, 608, 752

Bell, E. F., Naab, T., McIntosh, D. H., et al. 2005a, ApJ, submitted [arXiv: astro-ph/0506425]

Bell, E. F., Papovich, C., Wolf, C., et al. 2005b, ApJ, 625, 23

Benson, A. J., Ellis, R. S., \& Menanteau, F. 2002, MNRAS, 336, 564

Berta, S., Fritz, J., Franceschini, A., et al. 2004, A\&A, 418, 913

Bertelli, P., Bressan, A., Chiosi, C., Fagotto, F., \& Nasi, E. 1994, A\&AS, 106, 275

Bertin, E., \& Arnouts, S. 1996, A\&AS, 117, 393

Blanton, M. R., Hogg, D. W., Bahcall, N. A., et al. 2003, ApJ, 594, 186

Bolzonella, M., Miralles, J.-M., \& Pelló, R. 2000, A\&A, 363, 476

Bressan, A., Chiosi, C., \& Fagotto, F. 1994, ApJS, 94, 63

Bundy, K., Ellis, R. S., \& Conselice, C. J. 2005, ApJ, 625, 621

Calzetti, D., Armus, L., Bohlin, R. C., et al. 2000, ApJ, 533, 682

Cardelli, J. A., Clayton, G. C., \& Mathis, J. S. 1989, ApJ, 345, 245

Cassata, P., Cimatti, A., Franceschini, A., et al. 2005, MNRAS, in press

Chapman, S. C., Blain, A. W., Ivison, R. J., \& Smail, I. R. 2003, Nature, 422, 695

Chiosi, C., \& Carraro, G. 2002, MNRAS, 335, 335

Ciliegi, P., Zamorani, G., Hasinger, G., et al. 2003, A\&A, 398, 901

Cimatti, A., Daddi, E., Mignoli, M., et al. 2002a, A\&A, 381, L68

Cimatti, A., Pozzetti, L., Mignoli, M., et al. 2002b, A\&A, 391, 1

Cimatti, A., Daddi, E., Renzini, A., et al. 2004, Nature, 430, 184

Cole, A., Lacey, C. G., Baugh, C. M., \& Frenk, C. S. 2000, MNRAS, 319, 204

Cole, S., Norberg, P., \& Baugh, C. M. 2001, MNRAS, 326, 255

Conselice, C. J., Bershady, M. A., \& Jangren, A. 2000, ApJ, 529, 886

Conselice, C. J. 2003a, ApJS, 147, 1

Conselice, C. J., Bershady, M. A., Dickinson, M., \& Papovich, C. 2003b, AJ, 126,1183

Cowie, L. L., Songaila, A., Hu, E. M., \& Cohen, J. G. 1996, AJ, 112, 839

Croton, D. J., Ferrar, G. R., Norberg, P., et al. 2005, MNRAS, 356, 1155

Daddi, E., Cimatti, A., \& Renzini, A. 2000, A\&A, 362, L45

Daddi, E., Cimatti, A., Renzini, A., et al. 2004a, ApJ, 600, L127

Daddi, E., Cimatti, A., Renzini, A., et al. 2004b, ApJ, 617, 746

Daddi, E., Renzini, A., Pirzkal, N., et al. 2005a, ApJ, 626, 680

Daddi, E., Dickinson, M., Chary, R., et al. 2005b, ApJ, 631, L13

Dickinson, M., Papovich, C., Ferguson, H. C., \& Budavari, T. 2003, ApJ, 587, 25

Dickinson, M., et al. 2004, A\&AS, 204, 3313

Drory, N., Bender, R., Feulner, G., et al. 2004, ApJ, 608, 742

Eggen, O. J., Lynden-Bell, D., \& Sandage, A. R. 1962, ApJ, 136, 748

Elbaz, D., Cesarsky, C. J., Chanial, P., et al. 2002, A\&A, 384, 848

Ellis, R. 1997, ARA\&A, 35, 389

Faber, S., et al. 2005 [arXiv: astro-ph/0506044]

Fazio, G. G., Ashby, M. L. N., Barmby, P., et al. 2004, ApJS, 154, 39

Ferland, G. J. 1996, Hazy, a Brief Introduction to CLOUDY, in University of

Kentucky, Department of Physics and Astronomy Internal Report

Fontana, A., Pozzetti, L., Donnarumma, I., et al. 2004, A\&A, 424, 23

Franceschini, A., Danese, L., Toffolatti, L., \& de Zotti, G. 1988, MNRAS, 233, 157

Franceschini, A., Mazzei, P., De Zotti, G., \& Danese, L. 1994, ApJ, 427, 140

Franceschini, A., Silva, L., Fasano, G., et al. 1998, ApJ, 506, 600

Franceschini, A., Hasinger, G., Miyaji, T., \& Malquori, D. 1999, MNRAS, 310, L5

Franceschini, A., Aussel, H., Cesarsky, C. J., Elbaz, D., \& Fadda, D. 2001, A\&A, 378,1

Franceschini, A., Manners, J., Polletta, M., et al. 2005, AJ, 129, 2074

Franx, M., Labbé, I., Rudnick, G., et al. 2003, ApJ, 587, L79

Gavazzi, G., Pierini, D., \& Boselli, A. 1996, A\&A, 312, 397

Genzel, R., Lutz, D., Sturm, E., et al. 1998, ApJ, 498, 579
Giavalisco, M., Ferguson, H. C., Koekemoer, A. M., et al. 2004, ApJ, 600, 93 Glazebrook, K., Abraham, R. G., McCarthy, P. J., et al. 2004, Nature, 430, 181 Gruppioni, C., Lari, C., Pozzi, F., Zamorani, G., et al. 2002, MNRAS, 335, 831 Hasinger, G., Miyaji, T., \& Schmidt, M. 2005, MNRAS, 441, 417 Hatton, S., Devriendt, J. E. G., Ninin, S., et al. 2003, MNRAS, 343, 75 Hatziminaoglou, E., Cassata, P., Rodighiero, G., et al. 2005, A\&A, 364, 47 Hogg, D. W., Blanton, M., Strateva, I., et al. 2002, AJ, 124, 646 Ilbert, O., Tresse, L., \& Arnouts, S. 2004, MNRAS, 351, 541 Im, M., Simard, L., Faber, S. M., et al. 2002, ApJ, 571, 136 Ingber, L. 1989, Math. Comp. Model., 12, 967

Juneau, S., Glazebrook, K., Crampton, D., et al. 2005, ApJ, 619, 135

Kauffmann, G., Charlot, S., \& White, S. D. M. 1996, MNRAS, 283, 117

Kauffmann, G., \& Charlot, S. 1998, MNRAS, 297, L23

Kauffmann, G., Heckman, T. M., White, S. D. M., et al. 2003, MNRAS, 341, 33

Kennicutt, R. C., Jr. 1992, ApJ, 388, 310

Kennicutt, R. C., Jr. 1998, ARA\&A, 36, 189

Kitzbichler, M. G., \& White, S. D. M. 2004, MNRAS, submitted [arXiv: astro-ph/0409682]

Kochanek, C. S., Pahre, M. A., Falco, E. E., et al. 2001, ApJ, 560, 566

Krist, J. 2002, Tiny TIM/SIRTF User's guide, Spitzer Science Center internal document

Kurucz, R. L. 1993, ASPC, 44, 87

Labbé, I., Huang, J., Franx, M., et al. 2005, ApJ, 624, L81

Lagache, G., Dole, H., \& Puget, J. L. 2003, MNRAS, 338, 555L

Larson, R. 1975, MNRAS, 173, 671

Le Fèvre, O., Vettolani, G., Paltani, S., et al. 2004a, A\&A, 428, L1043

Le Fèvre, O., Mellier, Y., McCracken, H. J., et al. 2004b, A\&A, 417, L839

Le Floc'h, E., Papovich, C., Dole, H., et al. 2005, ApJ, 632, 169

Lilly, S. J., Le Fevre, O., Hammer, F., \& Crampton, D. 1996, ApJ, 460, L1

Longhetti, M., Bressan, A., Chiosi, C., \& Rampazzo, R. 2000, A\&AS, 353, 917

Lonsdale, C., Smith, H. E., Rowan-Robinson, M., et al. 2004, ApJS, 154, L54

Madau, P., Ferguson, H. C., Dickinson, M. E., et al. 1996, MNRAS, 283, 1388

Madau, P., \& Pozzetti, L. 2000, MNRAS, 312, 9

Mignoli, M., Cimatti, A., Zamorani, G., et al. 2005, A\&A, 437, 883

Mihos, J. C., \& Hernquist, L. 1994, ApJ, 437, L47

Mo, H. J., Mao, S., \& White, S. D. M. 1998, MNRAS, 295, 319

Mobasher, B., Idzi, R., Benytez, N., et al. 2004, ApJ, 600, L167

Mushotzky, R. F., \& Loewenstein, M. 1997, ApJ, 481, L63

Papovich, C., Giavalisco, M., Dickinson, M., Conselice, C. J., \& Ferguson, H. C. 2003, ApJ, 598, 827

Perez-Gonzalez, P., Rieke, G. H., Egami, E., et al. 2005, ApJ, 630, 82

Pickles, A. J. 1998, PASP, 110, 863

Poggianti, B., Bressan, S., \& Franceschini, A. 2001, ApJ, 550, 195

Rigopoulou, D., Spoon, H. W. W., Genzel, R., et al. 1999, AJ, 118, 2625

Rodighiero, G., Franceschini, A., \& Fasano, G. 2001, MNRAS, 324, 491

Rodighiero, G., Lari, C., Fadda, D., et al. 2004, A\&A, 427, 773

Rodighiero, G., Fadda, D., Lari, C., \& Franceschini, A. 2005, MNRAS, 357, 449

Rowan-Robinson, M., Mann, R. G., Oliver, S. J., et al. 1997, MNRAS, 289, 482

Rowan-Robinson, M. 2001, ApJ, 549, 745

Rowan-Robinson, M., Babbedge, T., Surace, J., et al. 2005, AJ, 129, 1183

Scalo, J. M. 1986, Fund. Cosmic Phys., 11, 1

Scoville, N., et al. 2004, A\&AS, 205, 7201

Schiminovich, D., Ilbert, O., Arnouts, S., et al. 2005, ApJ, 619, 47

Schmidt, M. 1968, ApJ, 151, 393

Silva, L., De Zotti, G., Granato, G. L., Maiolino, R., \& Danese, L. 2005, MNRAS, 357, 1295

Simpson, C., \& Eisenhardt, P. 1999, PASP, 111, 691

Smail, I., Ivison, R. J., Blain, A. W., \& Kneib, J. P. 2002, MNRAS, 331, 495

Somerville, R. S., \& Primack, J. R. 1999, MNRAS, 310, 1087

Somerville, R. S., Primack, J. R., \& Faber, S. M. 2001, MNRAS, 320, 504

Somerville, R. S., Moustakas, L. A., Mobasher, B., et al. 2004, ApJ, 600, L135

Springel, V., Di Matteo, T., \& Hernquist, L. 2005, MNRAS, 361, 776

Strateva, I., Ivezic, Z., Knapp, G. R., et al. 2001, AJ, 122, 1861

Sutherland, W., \& Saunders, W. 1992, MNRAS, 259, 413

Szeifert, T., et al. 1998, SPIE Proc., 3355

Szokoly, G. P., Bergeron, J., Hasinger, G., et al. 2004, ApJS, 155, 271

Thomas, D., Maraston, C., Bender, R., \& Mendes de Oliveira, C. 2005, ApJ, 621, 673

Tran, K. H., van Dokkum, P., Franx, M., et al. 2005, ApJ, 627, 25

Treu, T., Ellis, R. S., Liao, T. X., et al. 2005a, ApJ, 633, 174

Treu, T., Ellis, R., Liao, T., \& van Dokkum, P. 2005b, ApJ, 622, L5

Vanzella, E., Cristiani, S., Dickinson, M., et al. 2005, A\&A, 434, 53

Windhorst, R. A., Taylor, V. A., Jansen, R. A., et al. 2002, ApJS, 143, 113

White, S. D. M., \& Rees, M. J. 1978, MNRAS, 183, 341

White, S. D. M., \& Frenk, C. S. 1991, ApJ, 379, 52

Wolf, C., Meisenheimer, K., Kleinheinrich, M., et al. 2004, A\&A, 421, 913

Xu, C. K., Lonsdale, C. J., Shupe, D. L., et al. 2003, ApJ, 587, 90 